\title{
Identification of 1,531 cSNPs from Full-length Enriched cDNA Libraries of the Korean Native Pig Using in Silico Analysis
}

\author{
Younshin Oh, Dinh Truong Nguyen, Kwangha \\ Park, Vijaya R. Dirisala, Hojun Choi and \\ Chankyu Park*
}

Department of Animal Biotechnology, Konkuk University, Seoul 143-701, Korea

\begin{abstract}
Sequences from the clones of full-length enriched cDNA libraries serve as valuable resources for functional genomics related studies, genome annotation and SNP discovery. We analyzed 7,392 high-quality chromatograms (Phred value $\geq 30$ ) obtained from sequencing the $5^{\prime}$ ends of clones derived from full-length enriched cDNA libraries of Korean native pigs including brainstem, liver, cerebellum, neocortex and spleen libraries. In addition, 50,000 EST sequence trace files obtained from GenBank were combined with our sequences to identify cSNPs in silico. The process generated 11,324 contigs, of which 2,895 contigs contained at least one SNP and among them 610 contigs had a minimum of one sequence from Korean native pigs. Of 610 contigs, we randomly selected 262 contigs and performed in silico analysis for the identification of cSNPs. From the results, we identified 1,531 putative coding single nucleotide polymorphisms (cSNPs) and the SNP detection frequency was one SNP per $465 \mathrm{bp}$. A large-scale sequencing result of clones from full-length enriched cDNA libraries and identified cSNPs will serve as a useful resource to functional genomics related projects such as a pig HapMap project in the near future.
\end{abstract}

Keywords: cSNP, full-length cDNA library, functional genomics, Korean native pigs, pigs

\section{Introduction}

The pig, one of the fist animals domesticated over 7000 years ago, is the major meat supply resource consumed worldwise (Rothschild, 2003). Further more, the porcine has served as sensitive and comprehensive tools to address biomedical, agricultural and fundamental biological

${ }^{*}$ Corresponding author: E-mail chankyu@konkuk.ac.kr Tel +82-2-450-3697, Fax +82-2-457-8488 Accepted 9 June 2008 questions (Tuggle et al., 2007). To better comprehend the physiological complexity of the pig genome, a funtional gene analysis needs to be accomplished.

Single nucleotide polymorphisms (SNPs) are the most frequent form of DNA variations in the genome of organisms and useful for association studies, gene mapping and phylogenetic studies (Brookes, 1999; Dimmic et al., 2005; Kim, et al., 2007; Kim, et al., 2008; Komar, 2007). They provide genomic landmarks of past events shaping individuals, breeds and species (Lee et al, 2006a). Owing to high abundance and stability of SNPs in the genome, thousands of potentially informative SNP markers can be discovered and used to develop high-density SNP maps (Panitz et al., 2007; Zimdahl et al., 2004). These maps are an essential resource for the identification of genes that are responsible for variations in complex traits or quantitative trait loci (QTL; Glazier et al., 2002; Rothschild et al., 2007).

SNPs are classically identified by direct sequencing of amplicons of candidate genes from a set of individuals that represent the diversity in the interested populations. The draw backs of this approach are laborious and expensive (Useche et al, 2001). A fast and cost-efficient approach to identify a multitude of novel SNPs involving in mining sequence data from public repositories needed to be developed. The improvement of sequence trace data in public repositories at the DNA chromatogram level was required (Panitz et al., 2007).

An alternative and cheaper method of SNP identification exploits the redundancy of gene sequences generated by expressed sequence tags (ESTs; Gu et al., 1998). - In this scenario, each SNP would also be associated with an expressed gene. EST information has been used for the detection of SNPs in mammalian genomes by many groups (Buetow et al., 1999; Fitzsimmons et al., 2004; Garg et al., 1999; Guryev et al., 2004; Hawken et al., 2004; Kim et al., 2003; Lee et al., 2006a; Lee et al., 2006b; Picoult-Newberg, et al., 1999).

Many conventional cDNA libraries have been constructed with porcine material. As of May 2009, $1,532,429$ ESTs were available in the GenBank porcine EST database and 167,740 SNPs were achievable in the GenBank pig SNP database (Table 1). Several researchers have reported mining cSNPs from porcine EST sequences (Dirisala et al., 2005, 2007; Fahrenkrug et al., 2002; Grapes et al., 2006; Kollers et al., 2005; Panitz et al., 2007; Park et al., 2009; Uenishi et al., 
Table 1. The current status of reported SNPs from 6 animal species based on the dbSNP database at NCBI

\begin{tabular}{lcrr}
\hline \multicolumn{1}{c}{ Species } & dbSNP build & $\begin{array}{c}\text { No. of } \\
\text { submissions }\end{array}$ & $\begin{array}{r}\text { No. of RefSNP } \\
\text { clusters }\end{array}$ \\
\hline Bos taurus & 128 & $2,485,282$ & $2,457,983$ \\
Canis familiaris & 126 & $3,527,071$ & $3,301,322$ \\
Equus caballus & 128 & $1,163,580$ & $1,163,580$ \\
Homo sapiens & 130 & $79,485,538$ & $17,804,034$ \\
Mus musculus & 128 & $18,667,322$ & $14,380,528$ \\
Sus scrofa & 128 & $167,740^{2}$ & 8,424 \\
\hline
\end{tabular}

Data source: http://www.ncbi.nlm.nih.gov/projects/SNP/snp_ summary.cgi

${ }^{1}$ Reference SNP.

${ }^{2}$ Our new 1,529 cSNPs were not included.

2004). Recently, full-length enriched cDNA libraries have also been constructed (Chen et al., 2006; Dirisala et al., 2005; Fujisaki et al., 2004; Kim et al., 2006; Uenishi et al. 2007; Uenishi et al., 2004). Although the number of ESTs has increased significantly, less progress has been made in analyzing this large volume of emerging data, and a multitude of markers is necessary for the identification of economically important traits in pigs (Kollers et al., 2005).

A large-scale cSNP mining from EST sequences has been reported by the Sino-Danish pig genome sequencing initiative (Panitz et al., 2007), these cSNPs were analyzed using cDNA sequences constructed by conventional methods. However, sequences from full-length enriched cDNA libraries serve as more valuable resources for functional genomics studies than sequences from conventional cDNA libraries as they extend further towards the $5^{\prime}$ end and represent a critical tool for improving the quality of many genomic annotation parameters (Das et al., 2001; Haas et al., 2002). In spite of that, data from the analysis of full-length enriched cDNA libraries constructed using pig tissues and cells are still limited (Chen et al., 2006; Dirisala et al., 2005, 2007; Fujisaki et al., 2004; Kim et al., 2006; Uenishi et al., 2004, 2007).

In this report, we have evaluated the process of cSNP discovery using the five full-length enriched cDNA libraries of Korean native pigs from cerebellum, liver, brainstem, neocortex and spleen libraries as a diverse genetic resource. In the result, 1,531 new cSNPs from 262 contigs were identified from the in silico analysis of the sequences from these libraries and the trace data files from the public database.

\section{Methods}

\section{Tissue collection}

The Korean native pig is a medium-size pig with black coat color originating in the Korean peninsula (Kim et al., 2005). Neocortex, brainstem, liver, cerebellum, and spleen tissues were dissected from Korean native pigs that were maintained at the Jeju livestock experiment station in Jeju, Korea. The tissues were snap frozen in liquid nitrogen and stored at $-80^{\circ} \mathrm{C}$ until use.

\section{Construction of full-length enriched cDNA libra- ries}

The neocortex, cerebellum and spleen libraries were constructed using a SMART kit (Clontech Laboratories, Mountain View, CA, USA). The brainstem and liver library were constructed by the modified oligo-capping and modified vector-capping method (Dirisala et al., 2005 , 2007), respectively. Plasmid DNA was prepared in the 96-well plate format using multi-well filter plates (Pall Corporation, Greenvale, NY, USA).

\section{Sequencing of full-length enriched cDNA libra- ries}

Sequencing of cloned cDNA inserts was performed using an ABI PRISM Bigdye Terminator Cycle Sequencing Ready Reaction kit (Applied Biosystems, Foster City, CA, USA) according to the manufacturer's instructions, and resultant sequences were analyzed on ABI 3700 automated sequencers.

\section{SNP identification}

The 7,392 EST sequences with high-quality chromatograms were transferred into the Linux workstation. The method for bioinformatic analysis of full-length cDNA clones were performed as previously described (Park et al., 2009). Briefly, EST sequence trace files were base called using Phred (Ewing \& Green, 1998a; Ewing et al., 1998b) with the options trim_alt and trim_phd. Sequences assessed for having a Phred quality score higher than 30 in $20 \mathrm{bp}$ windows were subjected to Cross_match (P. Green, Unpublished) for vector trimming. RepeatMasker was used for removing repeat sequences (http://repeatmasker.genome.washington. edu/; A.F.A. Smit and P. Green, Unpublished). The processed sequences were assembled into contigs by Phrap (http://bozeman.mbt.washington.edu/phrap.docs/ phrap.html), integrated with Polyphred (http://droog.mbt. washington.edu/PolyPred.html) for finding SNP candi- 
date sites and viewed with the Consed program (http://bozeman .mbt. washington .edu/cosed/Consed.html) using the polymorphism option (Gordon et al., 1998). The polymorphic sites were identified, and the results were confirmed in individual chromatograms. The new cSNPs were determined by comparing our data with NCBI BLAST (http://www.ncbi.nlm.nih.gov/BLAST/) and the previously published paper (Park et al., 2009).

\section{Results}

\section{Characteristics of cerebellum and liver libraries}

Library titers for the cerebellum and liver libraries were $5.0 \times 10^{5} \mathrm{pfu}$ and $6.5 \times 10^{4} \mathrm{cfu}$, respectively. The average cDNA insert size estimated by restriction analysis of 20 clones picked up randomly from each library was $1.7 \mathrm{~kb}$ and $1.1 \mathrm{~kb}$ for the cerebellum and liver libraries, respectively. The analysis of other characteristics of libraries was performed as previously described (Dirisala et al., 2005, 2007).

A total of the 7,392 clones from five full-length enriched libraries $(4,416$ from cerebellum, 288 from liver, 1,056 from brainstem, 672 from spleen, 960 from neocortex) randomly picked into 96 well plates was sequenced from the only $5^{\prime}$ end direction. Although it is debatable to set the criteria for full-length clones, we used the presence of translation initiation codon 'ATG' in the cDNA sequences as the definition. According to our definition, the percentage of full-length clones was estimated to be $50 \%$ and $75 \%$ for the cerebellum and the liver libraries, respectively (data not shown). The percentage of full length clones for neocortex, brain stem and spleen was $40 \%, 80 \%$ and $60 \%$, respectively (Dirisala et al., 2005, 2007).

Since these libraries were specifically constructed for generating full-length clones, $65 \%$ of clones had longer $5^{\prime}$ end sequences than currently available EST sequences in the GenBank EST database. To further eval-

Table 2. The functional characteristics of contigs used for SNP discovery using bioinformatic analysis from cerebellar, liver, neocortex, brainstem and spleen cDNA libraries

\begin{tabular}{lc}
\hline \multicolumn{1}{c}{ Blast match } & No. of clones (\%) \\
\hline Non redundant $(\mathrm{nr})$ & $506(83.0)$ \\
Pig & $208(41.1)$ \\
Mitochondrial transcripts & $13(2.6)$ \\
Genomic sequence & $22(4.3)$ \\
Other species & $263(52.0)$ \\
EST & $93(15.2)$ \\
Non & $11(1.8)$ \\
\hline Total & $610(100)$ \\
\hline
\end{tabular}

uate the characteristics of five libraries, 610 contigs containing at least one sequence of the Korean native pig was selected and analyzed (Table 2). The result showed that $98 \%$ of sequences were matched with sequences of either pigs or other species with an $E$ value $<10^{-100}$.

\section{Identification of putative csnps using a bioinfor- matics approach}

We analyzed cDNA clones from five libraries where the cerebellar and liver libraries were constructed in this study and the neocotex, spleen and brainstem libraries were from our previous study (Dirisala et al., 2005, 2007) by $5^{\prime}$ end sequencing, and a total of 7,392 high-quality chromatograms (Phred value $\geq 30$ ) were obtained from sequencing the $5^{\prime}$ ends of clones. Furthermore, 50,000 chromatograms of porcine EST sequences were retrieved from the GenBank trace file archive, combined with the sequencing results from the cDNA libraries and analyzed for the presence of cSNPs.

The process yielded a total of 11,324 contigs, and 2,895 out of 11,324 contigs had cSNPs (data not shown). As we were interested in identifying cSNPs using Korean native pig sequences, only 610 contigs which had overlapping regions contributed by at least one sequence each from GenBank and Korean native pig were chosen for further analysis. The remaining contigs assembled only with Genbank sequences were excluded from our analysis. Of 610 contigs, only a part of contigs (262) were randomly selected for the discovery of cSNPs in this study due to the limitation from the manual confirmation of generated data through bioinformatic analysis. The analysis yielded the discovery of 1,533 cSNPs including 2 previously reported among the contigs containing Korean native pig sequences.

We analyzed the contribution in number of cDNA sequences for each contig used in cSNP discovery (Table 3). A total of 59 contigs were consisted of both one Korean native pig and one GenBank sequences which is the most abundant type. Out of 610 contigs, 182 were from the group with one Korean native pig sequence and at least one GenBank sequence. One hundred three contigs contained two Korean native pig sequences and at least one public EST sequence.

\section{Discussion}

Our primary goal for this study was to discover new cSNPs for pigs and to test the efficiency of cSNP identification by mixing CDNA sequences of full-length enriched cDNA libraries constructed from Korean native pigs and GenBank sequences. The application of our 
Table 3. The pattern of contribution in number of sequences for 610 contigs used for cSNP discovery from Korean native pigs and GenBank sequences

\begin{tabular}{|c|c|c|c|c|c|c|c|c|c|c|c|c|c|}
\hline \multirow{2}{*}{$\begin{array}{l}\text { No. of } \mathrm{KNP}^{1} \\
\text { sequences }\end{array}$} & \multicolumn{12}{|c|}{ NO. of GenBank sequences } & \multirow{2}{*}{ Total } \\
\hline & 0 & 1 & 2 & 3 & 4 & 5 & 6 & 7 & 8 & 9 & 10 & Others & \\
\hline 1 & - & $59^{2}$ & $36^{2}$ & $26^{2}$ & $15^{2}$ & 17 & 17 & 6 & 1 & 5 & - & - & 182 \\
\hline 2 & $25^{2}$ & $21^{2}$ & $17^{2}$ & $9^{2}$ & 8 & 12 & 3 & 4 & 4 & - & - & - & 103 \\
\hline 3 & $9^{2}$ & $14^{2}$ & $7^{2}$ & 6 & 2 & 5 & 1 & 1 & - & - & - & - & 45 \\
\hline 4 & $11^{2}$ & $10^{2}$ & 4 & 2 & 2 & 2 & 2 & - & - & - & - & - & 33 \\
\hline 5 & $3^{2}$ & 6 & 3 & 2 & 2 & 3 & - & - & - & - & - & - & 19 \\
\hline 6 & 4 & 3 & 4 & 3 & 3 & - & - & - & - & - & - & - & 17 \\
\hline 7 & 2 & 0 & 0 & 2 & - & - & - & - & - & - & - & - & 4 \\
\hline 8 & 3 & 1 & 0 & - & - & - & - & - & - & - & - & - & 4 \\
\hline 9 & 3 & 0 & - & - & - & - & - & - & - & - & - & - & 3 \\
\hline 10 & 2 & - & - & - & - & - & - & - & - & - & - & - & 2 \\
\hline Others & - & - & - & - & - & - & - & - & - & - & - & $198^{3}$ & 198 \\
\hline Total & 62 & 114 & 71 & 50 & 32 & 39 & 23 & 11 & 5 & 5 & - & 198 & 610 \\
\hline
\end{tabular}

${ }^{1}$ The KNP is Korean native pig.

${ }^{2}$ Contigs used for the discovery of 1,529 cSNPs by in sillico analysis.

${ }^{3} 198$ contigs were consisted of more than 10 sequences per contig.

study using the cDNA sequences of Korean native pigs as a source of genetic diversity in the porcine genome can provide additional resources for the identification of cSNPs. The genetic relationship between Korean native pigs and other pig breeds was expected to be rather distant (Porter, 1993). Thus, we predicted that there would be a higher possibility of identifying genetic differences between Korean native pigs and other breeds than between the commonly used breeds alone. The use of full-length enriched cDNA libraries also allowed to detect SNPs in the promoter regions of genes (Dirisala et al., 2005, 2007; Park et al., 2009)

The observed differences in SNP detection frequency arising from these reports might reflect genetic diversity within species and/or differences in application methods. In humans, the SNP detection frequency is reported to be about 1 SNP per 700 bp across the whole genome (The International SNP Map Working Group, 2001), and in some regions it is as high as 1 SNP per 300 bp (Wang et al., 1998). In chickens, the frequency of putative SNPs was variously reported to be 1 SNP per 2,119 bp (Kim et al., 2003) to 1 SNP per 1,900 bp (Fitzsimmons et al., 2004). In Korean native cattle, the SNP detection frequency of 1 SNP per 787 bp has been reported (Lee et al., 2006b). In our previous study, in which a small number of Korean native pig sequences and a relatively high stringency were used in selecting the candidate sequences, an in silico method yielded 1 SNP per 614 bp (Park et al., 2009). The results are very similar to our results in this study.

The removal of false SNPs from a large cSNP dataset generated from a large scale cDNA sequencing project is a difficult process since the most reliable method for the evaluation of SNPs is experimental confirmation based on animals genotyping against genetically diverse populations. One possible idea to solve this problem is to exploit the redundancy of CSNPs with existing EST sequences. If we can develop an algorithm to select the SNPs from polymorphisms existing in multiple times in the public database and evaluate some statistical values for the probability, it will greatly help to utilize this type of in silico approach for SNP discovery.

\section{Acknowledgements}

This work was supported by grants from the Technology Development Program for Agriculture and Forestry, Ministry for Agriculture, Forestry and Fisheries and the BioGreen21 program (20070401-034-029-010), Rural Development Administration, Republic of Korea.

\section{References}

Barker, G., Batley, J., Sullivan, H.O., Edwards, K.J., and Edwards, D. (2002). Redundancy based detection of sequence polymorphisms in expressed sequence tag data using autoSNP. Bioinformatics 19, 421-422.

Brookes, A.J. (1999). The essence of SNPs. Gene 234, 177-186.

Buetow, K.H., Edmonson, M.N., and Cassidy, A.B. (1999). Reliable identification of large number of candidate SNPS from public EST data. Nat. Genet. 21, 323-325.

Chen, C.H., Lin, E.C., Cheng, W.T.K., Sun, H.S., Mersmann, 
H.J., and Ding, S.T. (2006). Abundantly expressed genes in pig adipose tissue: an expressed sequence tag approach. J. Anim. Sci. 84, 2673-2683.

Dimmic, M.W., Sunyaev, S., and Bustamante, C. (2005). Inferring SNP function using evolutionary, structural and computational methods. Pac. Symp. Biocomput. 10, 382-384.

Dirisala, V.R., Kim, J., Park, K., Kim, N., Lee, K.T., Oh, S.J., Oh, J.H., Kim, N.S., Um, S.J., Lee, H.T., Kim, K.I., and Park, C. (2005). cSNP mining from full-length enriched cDNA libraries of the Korean native pig. Kor. J. Genet. 27, 329-335.

Dirisala, V.R., Kim, J., Park, K., Lee, H.T., and Park, C. (2007). Discovery of cSNPs in Pig Using Full-length Enriched cDNA Libraries of the Lorean Native Pig as a source of Genetic Diversity. BBE. 12, 424-432

Ewing, B., and Green, P. (1998a). Base calling of automated sequencing tracers using phred. II. Error probabilities. Genome. Res. 8, 186-194.

Ewing, B., Hillier, L., Wendl, M., and Green, P. (1998b). Base-calling of automated sequencer traces using Phred. I. Accuracy assessment. Genome. Res. 8, 175-185.

Fahrenkrug, S.C., Freking, B.A., Smith, T.P.L., Rohrer, G.A., and Keele, J.W. (2002). Single nucleotide polymorphism (SNP) discovery in porcine expressed genes. Anim. Genet. 33, 186-195.

Fitzsimmons, C.J., Savolainen, P., Amini, B., Hjalm, G., Lunderberg, J., and Andersson, L. (2004). Detection of sequence polymorphisms in red junglefowl and white leghorn ESTs. Anim. Genet. 35, 391-396.

Fujisaki, S., Sugiyama, A., Eguchi, T., Watanabe, Y., Hiraiwa, H., Honma, D., Saito, T., and Yasue, H. (2004). Analysis of a full-length cDNA library constructed from swine olfactory bulb for elucidation of expressed genes and their transcription initiation sites. J. Vet. Med. Sci. 66, 15-23.

Garg, K., Green, P., and Nickerson, D.A. (1999). Identification of candidate coding region single nucleotide polymorphisms in 165 human genes using assembled expressed sequence tags. Genome Res. 9, 1087-1092.

Glazier A.M., Nadeau J.H., and Aitman, T.J. (2002). Finding genes that underlie complex traits. Science 298, 2345-2349.

Gordon, D., Abajian, C., and Green, P. (1998). Consed: a graphical tool for sequence finishing. Genome Res. 8, 195-202.

Grapes, L., Rudd, S., Fernando, R.L., Megy, K., Rocha, D., and Rothschild, M.F. (2006). Prospecting for pig single nucleotide polymorphisms in the human genome: have we struck gold? J. Anim. Breed. Genet. 123, 145-151.

Gu, Z., Hillier, L., and Kwok, P.Y. (1998). Single-nucleotide polymorphism hunting in cyberspace. Hum. Mutat. 12, 221-225.

Guryev, V., Berezikov, E., Malik, R., Plasterk, R.H., and Cuppen, E. (2004). Single nucleotide polymorphisms associated with rat expressed sequences. Genome Res. 14, 1438-1443.

Hawken, R.J., Barris, W.C., McWilliam, S.M., and Dalrymple, B.P. (2004). An interactive bovine in silico SNP data- base (IBISS). Mamm. Genome 15, 819-827.

Kim, H., Shmidt, C.J., Decker, K.S., and Emara, M.G. (2003). A double-screening method to identify reliable candidate non-synonymous SNPs from chicken EST data. Anim. Genet. 34, 249-254.

Kim, J.H., Yim, S.H., Jeong, Y.B., Jung, S.H., Xu, H.D., Shin, S.H., and Chung, Y.J. (2008). Comparison of Normalization Methods for Defining Copy Number Variation Using Whole-genome SNP Genotyping Data. $G \& / 6,231-234$.

Kim, T.H., Kim, K.S., Choi, B.H., Yoon, D.H., Jang, G.W., Lee, K.T., Chung, H.Y., Lee, H.Y., Park, H.S., and Lee, J.W. (2005). Genetic structure of pig breeds from Korea and China using microsatellite loci analysis. J. Anim. Sci. 83, 2255-2263

Kim, T.H., Kim, N.S., Lim, D., Lee, K.T., Oh, J.H., Park, H.S., Jang, G.W., Kim, H.Y., Jeon, M., Choi, B.H., Lee, H.Y., Chung, H.Y., and Kim, H. (2006). Generation and analysis of large-scale expressed sequence tags (ESTs) from a full-length enriched cDNA library of porcine backfat tissue. BMC Genomics 7, 36.

Kim, Y.H., and Kim, H. (2007). Application of Random Forests to Association Studies Using Mitochondrial Single Nucleotide Polymorphisms. G\&/ 5, 168-173.

Kollers, S., Megy, K., and Rocha, D. (2005). Analysis of public single nucleotide polymorphisms in commercial pig populations. Anim. Genet. 36, 426-431.

Komar, A.A. (2007). SNPs, silent but not invisible. Science 315, 466-467.

Lee, M.A., Keane, O.M., Glass, B.C., Manley, T.R., Cullen, N.G., Dodds, K.G., McCulloh, A.F., Morris, C.A., Schreiber, M., Warren, J., Zadissa, A., Wilson T., and McEwan, J.C. (2006a). Establishment of a pipeline to analyse non-synonymous SNPs in Bos taurus. BMC Genomics 7 , 298.

Lee, S.H., Park, E.W., Cho, Y.M., Lee, J.W., Kim, H.Y., Lee, J.H., Oh, S.J., Cheon, I.C., and Yoon, D.H. (2006b). Confirming single nucleotide polymorphisms from expressed sequence tag datasets derived from three cattle cDNA libraries. J. Biochem. Mol. Biol. 39, 183-188.

Panitz, F., Stengaard, H., Hornshøj, H., Gorodkin, J., Hedegaard, J., Cirera, S., Thomsen, B., Madsen, L.B., Høj, A., Vingborg, R.K., Zahn, B., Wang, X., Wang, X., Wernersson, R., Jørgensen, C.B., Scheibye-Knudsen, K., Arvin, T., Lumholdt, S., Sawera, M., Green, T., Nielsen, B.J., Havgaard, J.H., Brunak, S., Fredholm, M., Bendixen, C. (2007). SNP mining porcine ESTs with MAVIANT, a novel tool for SNP evaluation and annotation. Bioinformatics 23, i387-i391.

Park, K., Dirisala, V.R., Oh, Y., Choi, H., Lee, K.T., Kim, J.H., Lee, H.T., Seo, K.H., and Park, C. (2009). Reporting 678 putative cSNPs from full-length enriched cDNA sequences of the Korean native pig. J. Anim. Breed Genet. 126(2), 127-133.

Picoult-Newberg, L., Idekar, T.E., Pohl, M.G., Taylor, S.L., Donaldson, M.A., Nickerson, D.A., and Boyce-Jacino, M. (1999). Mining SNPs from EST databases. Genome Res. 9, 167-174.

Porter, V. (1993) Pigs, A Handbook to the Breeds of the 
70 Genomics \& Informatics Vol. 7(2) 65-84, June 2009

World. Helm information Ltd., UK.

Rothschild, M.F. (2003). Advances in pig genomics and functional gene discovery. Comp. Funct. Genom. 4, 266-270.

Rothschild, M.F., Hu, Z.L., and Jiang, Z. (2007). Advances in QTL Mapping in pigs. Int. J. Biol. 3, 192-197

Sambrook, J., Fritsch, E., and Maniatis, T. (1989). Molecular cloning: A laboratory manual, 2nd ed., Cold Spring Harbor Laboratory Press, Cold Spring Harbor, NY, USA.

The International SNP Map Working Group. (2001). A map of human genome sequence variation containing 1.42 million single nucleotide polymorphisms. Nature 409, 928-933.

Tuggle, C.K., Wang, Y., and Couture, O. (2007). Advances in Swine Transcriptomics. Int. J. Biol. Sci. 3, 132-152

Uenishi, H., Eguchi, T., Suzuki, K., Sawazaki, T., Toki, D., Shinkai, H., Okumura, N., Hamasina, N., and Awata, T. (2004). PEDE (Pig EST Data Explorer): Construction of a database for ESTs derived from porcine full-length cDNA libraries. Nucl. Acids Res, 32, 484-488.

Uenishi, H., Eguchi-Ogawa, T., Shinkai, H., Okumura, N., Suzuki, K., Toki, D., Hamasima, N., and Awata, T. (2007). PEDE (Pig EST Data Explorer) has been expanded into pig expression data explorer, including 10147 porcine full-length cDNA sequences. Nucleic Acids Res. 35, D650-D653.

Useche, F.J., Gao, G., Harafey, M., and Rafalski, A. (2001). High-throughput identification, database storage and analysis of SNPs in EST sequences. Genome Inform. Ser. Workshop Genome Inform. 12, 194-203.

Wang, D.G., Fan, J.B., Siao, C.J., Berno, A., Young, P., Sapolsky, R., Ghandour, G., Perkins, N., Winchester, E., Spencer, J., Krugylyak, L., Stein, L., Hsie, L., Topaloglou, T. Hubbell, E., Robinson, E., Mittmann, M., Morris, M.S., Shen, N., Kilburn, D., Rioux, J., Nusbaum, C., Rozen, S., Hudson, T.J., and Lander, E.S. (1998). Large-scale identification, mapping, genotyping of single nucleotide polymorphisms in the human genome. Science 280, 1077-1082.

Zimdahl, H., Nyakatura, G., Brandt, P., Schulz, H., Hummel, O., Fatmann, B., Brett, D., Droege, M., Monti, J., Lee, Y.A., Sun, Y., Zhao, S., Winter, E.E., Pontig, C.P., Chen, Y., Kasprzyk, A., Birney, E., Ganten, D., and Hubner, N. (2004). A SNP map of rat genome generated from cDNA sequences. Science 303, 807. 
Table S1. The list of 1,529 new cSNPs identified from in silico analysis

\begin{tabular}{|c|c|c|c|}
\hline $\begin{array}{l}\text { Acc. No. of } \\
\text { blast match }\end{array}$ & Description & e-value & Sequence variation \\
\hline EU650787 & $\begin{array}{l}\text { hydroxyacyl-Coenzyme A } \\
\text { dehydrogenase/3-ketoacyl-Coenzyme } \\
\text { A thiolase/enoyl-Coenzyme A } \\
\text { hydratase (trifunctional protein), alpha } \\
\text { subunit }\end{array}$ & 0 & AAAGAAAATGGG (G/A) CTGGTTGACC \\
\hline AY996813 & RAB1A, member RAS oncogene family & 0 & $\begin{array}{l}\text { CA (A/G) ATATGGGACACAGCAGGCCA (A/G) GAA } \\
\text { CAATCACCT (C/A) CAG (T/C) TACTA(C/T) AGAGG }\end{array}$ \\
\hline BC009018 & enolase 2, gamma neuronal & 0 & CCGTGGGAATC $(\mathrm{C} / \mathrm{T})$ CACAGTGGA \\
\hline XM_001925729 & similar to Gamma-enolase & 0 & $\begin{array}{l}\text { TGGTATCTATGA (A/G) GCCCTGGAGCTA } \\
\text { GTGGACCACATC (G/A) ACACCACCATCG } \\
\text { CTTTGCCCCCAA (C/T) ATCCTGGAGAATA }\end{array}$ \\
\hline XR_027670 & $\begin{array}{l}\text { similar to lipocalin-interacting } \\
\text { membrane receptor }\end{array}$ & 0 & $\begin{array}{l}\text { TCCTGCCC (C/T) TTCCATCA } \\
\text { CAACTTGTCCCTC(G/A) TCTTCCTCA }\end{array}$ \\
\hline BC103155 & stromal cell-derived factor 2 & 0 & GGGGGAAGAC (T/C) GCCACAGTGT \\
\hline BC112833 & phosphatidylserine synthase 1 & 0 & GCA (C/T) CTGGTTGGGCATGGTCGTCTG (T/C) CG \\
\hline XM_864974 & similar to cell adhesion molecule JCAM & $8 e-122$ & GAAAGGCACGG (T/G) GGTTCTGAGCGTCA \\
\hline EW070413 & $\begin{array}{l}\text { rduo412b_c14.y1 duo Sus scrofa cDNA } \\
5 \text { ', mRNA sequence }\end{array}$ & $5 e-111$ & CGGGAATTGA (C/T) GGGGATAGGG \\
\hline XM_001924706 & $\begin{array}{l}\text { similar to asparagine-linked } \\
\text { glycosylation } 14 \text { homolog }\end{array}$ & 0 & $\begin{array}{l}\text { TGAAC (T/C) CAATCGAGCTGATC (A/G) GAAC } \\
\text { CCGGGAGGTTCA (A/G) CAGTCCTGGCCGTCC }\end{array}$ \\
\hline BC151565 & intraflagellar transport 57 homolog & 0 & TATTATCTGAGCTT (C/T) GATCATTCGGGAGAAC \\
\hline AK225203 & $\begin{array}{l}\text { suppressor of variegation } 4-20 \text { homolog } \\
1\end{array}$ & 0 & CATAATAACTGTAG (G/A) AAGTGTACAGTGTACTGA \\
\hline XM_001113664 & $\begin{array}{l}\text { similar to single-stranded DNA-binding } \\
\text { protein isoform a }\end{array}$ & $2 e-138$ & $\begin{array}{l}\text { GCCGCCCCGAGCCCCGTGCT (A/T) GG (A/C) AACA } \\
\text { CCC (A/C) (G/A) (G/A) (A/C) GATGG (C/G) AT } \\
\text { C (A/G) G (T/G) AGG (T/C) CC (T/C) AT (C/A) C } \\
\text { C (A/G) GGGTTCTTTCAGCCTTTTATGTCACC (T/G) C }\end{array}$ \\
\hline XM_001167299 & spermatogenesis associated 2 & $8 e-112$ & ATGCCCACCTGGAG (T/C) GGCGAGCTAGAGGCC \\
\hline XM_908972 & $\begin{array}{l}\text { triple functional domain (PTPRF } \\
\text { interacting) }\end{array}$ & $6 e-126$ & $\begin{array}{l}\text { ACTCGCTGTC (T/C) CGAGACACTGCAGCGTCG } \\
\text { GCAGCACTGTGGCA (A/G) AAACCACGCTGGTG }\end{array}$ \\
\hline XM_001928320 & $\begin{array}{l}\text { similar to Brain and acute leukemia } \\
\text { cytoplasmic protein }\end{array}$ & 0 & $\begin{array}{l}\text { CCACACCCAC (G/A) TCTCTCTCCTCCACCTTCACC } \\
\text { GAATC (G/A) TAGCATCACAGTCA (C/T) GGGCAAT } \\
\text { GTGTTTCTCCAGCTTTCC (A/T) CTTCCTTTCCTTGTG } \\
\text { TTTCCTTGTGCTGAGC (G/A) TTTTTCTCTCCATTCA } \\
\text { AATCAAAGACC (G/A) GCCACTGCTCACAAGAGGGA } \\
\text { TTAGCCTCTCCTTGCACA (T/C) CCAGGACTGTGTCA }\end{array}$ \\
\hline BC114879 & $\begin{array}{l}\text { GIMAP4 I GTPase, IMAP family } \\
\text { member } 4\end{array}$ & $1 e-136$ & GCATCCTGGGAAAGA (G/A) AGTGTTTCCTTCCG \\
\hline XR_028356 & similar to zinc finger protein 650 & 0 & GCCCAGT (A/G) GTGGTGATCTTCATG \\
\hline BC142261 & transmembrane protein 223 & $2 e-164$ & TGCCGCCTTA (G/A) CCCGCCCCCCGG \\
\hline XM_580320 & STEAP family member 3 & $3 e-100$ & $\begin{array}{l}\text { GAGCGCATTA (T/C) GGTCCTTTAGAGAA } \\
\text { GGGCCCTGA (T/C) TGAGCGGCAGCTGTG }\end{array}$ \\
\hline XM_001914786 & autism susceptibility candidate 2 & 0 & TCCGAAACGGTAA (T/C) GGAGGACGTGAG \\
\hline $\mathrm{BC} 112491$ & glutathione S-transferase mu 3 & 0 & ATGAAGAGAAAC (A/G) GTACATCTGCGGG \\
\hline XM_001496172 & ARP6 actin-related protein 6 homolog & 0 & GACCCTTCTGGACTC (C/T) TTTATATCCTTCCT \\
\hline XR_045452 & similar to Copine VI & 0 & $\begin{array}{l}\text { GATGGCATGGG (T/C) GCCTGAGCCCCCAGCC } \\
\text { C (G/A) CTGGG (A/G) GCCTCG (C/A) GAGT (A/G) G } \\
\text { CTGCCACGGCCT (T/C) CT (A/C) GACCGAGAC } \\
\text { CC (T/C) CA (T/C) CCCTG (C/T) GTGCT (C/G) CT } \\
\text { CAAGCTCCACTC (T/C) GATGAGCAGTGGGTGGA } \\
\text { GA (T/G) CGCAC (G/A) GAGGTGCTGCGC (C/T) CC } \\
\text { GCCC (T/C) GTCTTCTCCCG (G/C) GT (G/A) CTGGC } \\
\text { CCTGGAGTA (T/C) TTCTT (T/C) GAGGAGAGCA } \\
\text { GCAGTTCCA (C/T) GTGTT (C/T) GACGCTGAGGA }\end{array}$ \\
\hline BC010357 & arginine/serine-rich coiled-coil 1 & 0 & CCAGGAAGGCGG (T/C) GAGCTTAAACTGAAGCA \\
\hline
\end{tabular}


72 Genomics \& Informatics Vol. 7(2) 65-84, June 2009

Table S1. Continued

\begin{tabular}{|c|c|c|c|}
\hline EF635914 & $\begin{array}{l}\text { COP9 constitutive photomorphogenic } \\
\text { homolog subunit } 4\end{array}$ & 0 & GATCTGGCGGGCAAGT (G/A) TCGTCAGATCCTAGAAA \\
\hline BC146134 & $\begin{array}{l}\text { chromosome } 21 \text { open reading frame } 51 \\
\text { ortholog }\end{array}$ & $4 e-122$ & $\begin{array}{l}\text { GGCCCGGAGCT (C/-) (A/-) (G/-) GACAGTGAGACCTGGC } \\
\text { TCAAGATATACC }(\mathrm{G} / \mathrm{A}) \text { AAGGAAAGATTGGAAGCAA }\end{array}$ \\
\hline EW172950 & $\begin{array}{l}\text { rfbs3932b_p13.y1 fbs Sus scrofa cDNA } \\
\text { 5', mRNA sequence }\end{array}$ & 0 & $\begin{array}{l}\text { TGAGCTCGTGAGA (C/T) GAGCTTTCCCCACT } \\
\text { CAGAAGGAAGTGAA (T/C) AGAAATTGATAACA } \\
\text { TCCCACTACCTTCC }(\mathrm{G} / \mathrm{A}) \text { TGTGCATCTCTGCC }\end{array}$ \\
\hline XM_001929242 & $\begin{array}{l}\text { similar to Uncharacterized protein } \\
\text { C9orf78 }\end{array}$ & 0 & AGAGGTACAGAA (T/C) TTGAGAAAGAGGCCCA \\
\hline AY610017 & $\begin{array}{l}\text { Sus scrofa clone } \\
\text { Clu_5617.scr.msk.p1.Contig3, mRNA } \\
\text { sequence }\end{array}$ & $1 e-117$ & $\begin{array}{l}\text { TTCACTC (C/T) TGCAC (T/A) GTG } \\
\text { ATTGCCACCAA (T/C) ATTCTCCTGGT } \\
\text { TGA (T/C) GA (G/A) AT (C/T) ATG (A/C) GAGCT } \\
\text { GG (A/T) ATGTCTTCT (C/T) TAAAAGG (T/G) TGAA }\end{array}$ \\
\hline AF380118 & selenoprotein $\mathrm{W}, 1$ & 0 & GCCGTTCGAGTCGTCTAT (C/T) GTGGCGCTTGAGGCTA \\
\hline BC114078 & $\begin{array}{l}\text { glutaminyl-tRNA synthase } \\
\text { (glutamine-hydrolyzing)-like } 1\end{array}$ & 0 & $\begin{array}{l}\text { GCGCAGCGGCCG }(\mathrm{C} / \mathrm{T}) \text { GTCAGCGTTCACC } \\
\text { CCAAGCTATGGCT }(\mathrm{C} / \mathrm{T}) \text { AGTGTCTCGTCATG }\end{array}$ \\
\hline XM_844642 & ataxin 2-like & 0 & $\begin{array}{l}\text { ATCGGCAGC (G/C) CC (A/C) GAGGACAAAGCAC } \\
\text { ACAGCTGTTGTGGGCTC (T/C) ACTTGTGATGTA } \\
\text { GCTGTGCACCGGAAAGC (C/A) TCTGAGCCA }\end{array}$ \\
\hline XM_584414 & superoxide dismutase 1 , soluble & 0 & ACGAACACTTGGA (G/A) GATGTGTATAATTT \\
\hline NM_004447 & $\begin{array}{l}\text { epidermal growth factor receptor } \\
\text { pathway substrate } 8\end{array}$ & $7 e-111$ & ATCCTGTGAAAGA (T/C) TCTAGGTAGAGAAA \\
\hline AK235024 & regulator of G-protein signaling 5 & 0 & TATTGACCACTTCAC (G/A) AAGGAGATCACAA \\
\hline XM_001141083 & F-box protein 5 & $4 e-180$ & AGGCTGTGGATTTGA (C/T) TATTGTACAAGGT \\
\hline XM_001100585 & schwannomin interacting protein 1 & 0 & GGCACTTGGAAGC (C/T) TCTTTGATGATGGC \\
\hline AK239800 & programmed cell death 10 & 0 & TGC (G/A) GACAATCAAGGATATAGCC (G/A) GTG \\
\hline AK231106 & similar to vacuolar protein sorting 26 & 0 & TTCAATGACAAGAGTA (G/A) TACTCACGAATT \\
\hline AK238862 & $\begin{array}{l}\text { similar to DEAD (Asp-Glu-Ala-Asp) box } \\
\text { polypeptide } 55\end{array}$ & 0 & CTGAAGGTTGGTAC (C/T) TTTATCCTTTGTCT \\
\hline XR_045734 & $\begin{array}{l}\text { similar to peptidylprolyl isomerase } \\
\text { (cyclophilin) -like } 1\end{array}$ & 0 & TCCCAAGACCGCCC (T/C) GTGGACGATGTG \\
\hline AK238476 & RAB32, member RAS oncogene family & 0 & $\begin{array}{l}\text { GTGTGAACCTTCC (T/G) GGGGCTGTGCCTT } \\
\text { AAAATGACAAGG (A/G) TGGCACCCCAGGA }\end{array}$ \\
\hline EF416571 & SPARC-like 1 & 0 & CTTAAGTTCCTT (C/T) CAGCAGGCTGAAACG \\
\hline NM_138962 & musashi homolog 2 & 0 & GTCACAA (G/A) AA (C/A) AAA (G/A) AAAAT \\
\hline XM_858153 & $\begin{array}{l}\text { protein kinase, cAMP-dependent, } \\
\text { regulatory, type II, alpha }\end{array}$ & $9 e-124$ & TGGCATATTCTTTG (A/G) GTAGCAGAGAGCC \\
\hline XM_001927219 & $\begin{array}{l}\text { similar to single-strand selective } \\
\text { monofunctional uracil DNA glycosylase }\end{array}$ & 0 & GCAATCTCACCCC (T/C) GCCGAACTGCCGGC \\
\hline BC102483 & abhydrolase domain containing 1 & 0 & СTCTTCССTGAACCC (A/C) CAGAATGACAGCT \\
\hline BC103326 & OCIA domain containing 2 & 0 & $\begin{array}{l}\text { GAAGAGAATCGAAGACAA (A/G) GTCA (C/T) CATG } \\
\text { CT (G/T) CAGT (A/G) TC (T/C) AC (T/A) (G/C) AT } \\
\text { G (G/A) AAACCAAGA (C/A) AAAG (C/G) TCCCCAT } \\
\text { GCCACCAC (T/C) (T/A) AGCAAGCAGAGTCTGTTGTT } \\
\text { AAA (G/A) CTGCACATCCA (T/C) AGA (G/T) CAGAG } \\
\text { GAT (A/T) ATCCGGGAATGTCAAGA (G/A) GAAAGTTT } \\
\text { TT (A/G) TCACCCAAGG (G/A) CTGGTCCA (C/T) CA TG } \\
\text { GCAGC (T/G) AACCCGAG (G/A) TTTGGATCATTG }\end{array}$ \\
\hline BC109995 & tetraspanin 31 & $2 e-168$ & AGACGGTCCCCG (C/G) GACCCTCCCCCCAA \\
\hline XR_045530 & $\begin{array}{l}\text { similar to topoisomerase (DNA) II beta } \\
180 \mathrm{kDa}\end{array}$ & 0 & $\begin{array}{l}\text { GTTTTATTTACC (C/T) GTTTCAAGAGGAATGAT } \\
\text { TGTGAATTTGGCTCA (A/G) AACTTTGTGGGAA }\end{array}$ \\
\hline BC103122 & $\begin{array}{l}\mathrm{SH} 3 \text { domain binding glutamic acid-rich } \\
\text { protein like }\end{array}$ & $1 e-168$ & ATTCTGAAAATTTC (C/T) AAGAAGACAAGAAAT \\
\hline XM_522913 & KIAA1737 & 0 & $\begin{array}{l}\text { TGGTTTAGATA (T/C) GACCA (A/G) TTTAGAAA } \\
\text { TTTGGCAGAATATG (C/T) TTGGCAGCAAAGCT }\end{array}$ \\
\hline AK235840 & paraoxonase 2 & 0 & TAAAGGAATTGAAG (T/C) CGGCTCTGAAGATA \\
\hline J03489 & dihydrolipoamide dehydrogenase & 0 & AAACACTTGGTGGA (G/A) CATGCTTGAATGTT \\
\hline
\end{tabular}


Table S1. Continued

\begin{tabular}{ll}
\hline AK232420 & alpha-1-antichymotrypsin 2 \\
& \\
& \\
BC148062 & $\begin{array}{l}\text { dynein, light chain, Tctex-type 3 } \\
\text { similar to Beta-2-glycoprotein I } \\
\text { XM_001499678 }\end{array}$ \\
AK094090 & $\begin{array}{l}\text { defects in morphology 1 homolog } \\
\text { ribosomal protein L26 }\end{array}$ \\
BC141993 &
\end{tabular}

\begin{tabular}{|c|c|}
\hline FD632396 & $\begin{array}{l}\text { susfleck_FC_N_14_E02 SUSFLECK Fat } \\
\text { Cell Normalized Sus scrofa cDNA } \\
\text { clone 14_E02, mRNA sequence }\end{array}$ \\
\hline BT020945 & BCS1-like \\
\hline AK239939 & cAMP-regulated phosphoprotein \\
\hline XM_001918249 & hypothetical LOC100056802 \\
\hline U49178 & protein kinase $\mathrm{C}$ substrate $80 \mathrm{~K}-\mathrm{H}$ \\
\hline XM_001914994 & hypothetical LOC100073052 \\
\hline XM_001926357 & similar to isocitrate dehydrogenase \\
\hline XM_001927037 & similar to Rraga protein \\
\hline BC149559 & $\begin{array}{l}\text { protein (peptidylprolyl cis/trans } \\
\text { isomerase) }\end{array}$ \\
\hline & NIMA-interacting, 4 \\
\hline BC022524 & fibroblast growth factor 12 \\
\hline XM_001928575 & similar to Nck-associated protein 1 \\
\hline XM_607424 & $\begin{array}{l}\text { zinc finger, } \mathrm{CCHC} \text { domain containing } \\
11\end{array}$ \\
\hline XM_001927393 & $\begin{array}{l}\text { similar to growth hormone inducible } \\
\text { transmembrane protein }\end{array}$ \\
\hline XM_516815 & $\begin{array}{l}\text { transmembrane } 4 L \text { six family member } \\
1\end{array}$ \\
\hline DQ104414 & small muscle protein, X-linked \\
\hline AK233760 & $\begin{array}{l}\text { similar to tetratricopeptide repeat } \\
\text { domain } 5\end{array}$ \\
\hline
\end{tabular}

0 GCAAGAACAGGACGGTGA (G/A) GGTGCCCATG ATG $(G / A)$ GCATT $(A / C) G(G / A) G(C / G)$ CCT $G(A / G)(C / A)(C / A)(G / A)(T / C)(G / A) C C(A / T)$ TA ATCCTCCCTGACGA (C/T) GGCAGG ATGG (A/C) GG (A/C) CGTGGAAG CAAG (T/C) TGCTCCC (T/G) GAG

0 AAATTTTCAGGCAT (C/T) ACCTGTATGTTACT

0 TTGAAATCATCCTA (C/T) GCGCCAGGGGAGG

5e-155 ACTGACCTCTGTA (T/C) TCAGAACTGGTGCG

0 GGT (T/G) GT (A/C) CGAGG (G/A) CACTA CAA (G/A) GG (G/T) CAGCA (G/A) ATTGG AAGTAGTCCAGGT (T/G) TACAGGAAGAAAT TCTACAT (T/C) GA (A/G) CG (A/G) GTGCAGC TAATGG (C/T) ACAACTGTCCA (T/C) GTGGGCA (C/G) AGCAAGGT (G/A) GTTATCAC (T/C) AG (A/G) A $(G / A)$ GA $(C / T)$ CG (C/G) AAAAA (G/A) AT (C/T) C TTGA (A/C) CG (T/C) AAAGCCAAATCTCG (C/A) CAA GT (A/T) GGAAA (G/A) GAAAA (G/A) GG (A/C) AAAT AGAAACCATTGAGAA (G/A) ATGCAGGAGTGAATATAC 0 ATGGACATTAAGATA (C/T) TGAATAGAGAAGA

0 ACTCAGCACCTCAG (C/T) GTCGAGACTTCGTA

0 AAGCGAGGTATCC (C/T) CATCTGGGACAAAAG CTGGTGACCACAT (C/T) CCCACTCCACAGGAC ATTAAAAGAGCTG (G/A) ACTGCATGAATCTTCT 0 CCCACGCGTCGCC (T/C) AGGACCCGCTCG CAGCACTACCTCC (T/G) CCGGACTACCGCC

0 AAGAAGGCCCGAG (G/A) GGAGAAGCAGAAA

0 CCTGGAAGGCCAG (T/G) ACGCGTTCTTCCTG

0 CTGGCAGTTCATCA (G/A) GGAGAAGCTCATC CTGCCCCACGTGG (G/A) TGTCCAGCTCAAGT

0 GGGCCCAGTCAGC (T/G) AGCCCCCGCGACTC GGGCAACCTGGTG (T/C) TGAACCTGTGGGAC GTTTCCGAACATCC (G/A) TCTGGGATGAAACG

0 CACCGAAAGGAAAA (G/A) GCGCATCGGGAAAA AAAAAGCCTCAGGG (T/C) CCCAAAGGTGGTGG ATTTCAAGAAGCAGC (G/A) TTCGCCTTGCCTAT

2e-146 TGTC (G/T) GCAGATTTATGGATAT (C/T) ATCA

0 CATACATTATCTC (C/T) ATGTTTTTGATATTTG TGTAACACTGTAT (T/A) ACATTAAAAAATGTTT

0 CT (G/T) TGGTTGGGACT (T/G) CTTCG (T/C) TTC TA $(C / T)$ AC (A/T) GA (G/A) GA (A/G) TTTGA TTT (T/C) AAAGA (A/G) (C/T) ATGT (T/A) ATTAG CAT (C/T) (A/C) GGCAGAAAAA (G/A) CTG (C/T) T (T/G) ACAACTTTT (A/G) AGAA (A/G) CAGTG GAC (C/T) TC (A/C) AA (G/A) T (A/G) (C/T) AT TG (T/C) (T/G) ATTGAAGATCC (A/T) TTTGAT AA (T/C) CTTGG (A/T) GCTGGA (T/G) TT (A/T) CT 0 GTGCCACCATGCT (G/A) GCGGCAAGACTCGTG

\section{2e-159 GACTGCTGTGGCTGCTG (C/T) GGCCATGAAA}

0 GGAGATCCAGAACAT (C/T) AAAAGTGAACTGA

0 GAAGCTGGAGCCCAA (G/A) CTGGTGGAAG TCAATGGTGCTTCG (C/T) CAGCTGCGGACT 
74 Genomics \& Informatics Vol. 7(2) 65-84, June 2009

Table S1. Continued

\begin{tabular}{|c|c|c|c|}
\hline XM_001927571 & $\begin{array}{l}\text { similar to PC4 and SFRS1-interacting } \\
\text { protein }\end{array}$ & 0 & САAACTCTACTAGTAA (C/A) TTACTTCTTTGC \\
\hline XR_045568 & $\begin{array}{l}\text { similar to Uncharacterized protein } \\
\text { KIAA1107 }\end{array}$ & 0 & $\begin{array}{l}\text { GCTGA (G/T) AGCTTAATA (T/C) TAGAAAA } \\
\text { TTAACTCTTTTTC (A/G) GATATATCCAGAGA }\end{array}$ \\
\hline AB000425 & neurolysin (metallopeptidase M3 family) & 0 & CTGTAAGAAGTG (T/C) GGCAAGCACCAAC \\
\hline XM_001141202 & $\begin{array}{l}\text { catenin (cadherin-associated protein), } \\
\text { delta } 1\end{array}$ & 0 & CTTTTGGGGACCT (T/A) TAACTTTTTTTTTT \\
\hline XM_001929509 & $\begin{array}{l}\text { similar to Mitogen-activated protein } \\
\text { kinase } 1\end{array}$ & 0 & GTCCCTGGCAGCCC (G/T) GTGTGTGTCAC \\
\hline XM_001500465 & $\begin{array}{l}\text { similar to template acyivating factor-I } \\
\text { alpha }\end{array}$ & 0 & AAAGGGTGGGTTTGA (A/G) ATGTTTGGGTGGGT \\
\hline XR_045658 & $\begin{array}{l}\text { similar to mitochondrial ribosomal } \\
\text { protein } \\
\text { S28 }\end{array}$ & 0 & $\begin{array}{l}\text { GAGTGTTTTCTTCTC (T/C) CGGCCCTGTAGAGG } \\
\text { AAATGTGGAGTCCTT (T/C) GCATCTATGCTGAGA }\end{array}$ \\
\hline BC142418 & NECAP endocytosis associated 1 & $3 e-175$ & $\begin{array}{l}\text { GT (C/T) CT (G/C) TGTGT (G/C) AAGCC (C/T) G } \\
\text { A (C/G) GTCAGCGTCTACCG (G/C) AT (T/C) CCG } \\
\text { CC (C/G) CGGGCC (T/A) CCAACCG (C/T) GGTTA } \\
\text { CAGGGC (A/T) TC (T/G) GA (C/G) TGG (A/C) A } \\
\text { (A/G) (T/C) T (A/G) GA (T/C) CA (A/G) CC } \\
\text { AT (G/C) ATGGA (C/G) (A/T) GG (T/C) C } \\
\text { G (C/G) CT (C/G) CG (A/G) ATCACT (T/G) } \\
\text { C (C/A) AAAGGG (A/C) AGGTGGC (G/C) T } \\
\text { A (T/C) ATCAA (A/G) CTCGAGGA (T/C) A } \\
\text { (A/G) (A/G) (G/A) (T/C) (T/C) TCAGGGGAG } \\
\text { C (A/C) CC (A/T) GT (A/G) GA (A/T) CA (A/G) T } \\
\text { (A/T) TCCTGG (T/C) A (T/C) (C/A) GC (T/G) GT } \\
\text { GGAGA (C/G) (A/T) GTG (G/A) C (A/G) GATTC } \\
\text { CAGC (C/A) G (C/G) TACT (T/C) GT (A/T) AT } \\
\text { CCG (G/C) AT (C/T) (C/G) A (G/A) GA (T/C) G } \\
\text { G(T/A) A (C/A) TGGGCG (C/A) (A/C) G (T/G) G } \\
\text { C (T/A) TT (C/T) ATTGG (C/A) AT (C/T) GGC } \\
\text { TTC (A/G) (C/G) (A/G) GA (T/C) CGAGGT } \\
\text { GA (T/C) GCCTT (C/T) GACTT (T/C) AATG } \\
\text { T (C/T) (T/G) C (T/C) TT (A/G) CA (A/G) GA } \\
\text { CCA (C/T) TTCAA (G/A) TGGGT (A/G) AAGC } \\
\text { AG (G/C) A (A/G) T (C/G) TGAGATTT (C/G) CA }\end{array}$ \\
\hline XM_534074 & similar to small acidic protein & 0 & CGATCTTGGA (C/T) CTAGCAATTGGGA \\
\hline BC116061 & breast cancer metastasis suppressor 1 & 0 & GGCAGCTCGA (G/A) GACCTGGGACTC \\
\hline AK234663 & RAB3A, member RAS oncogene family & 0 & $\begin{array}{l}\text { ACTACATGTT (T/C) AAG (A/C) TCCTCAT } \\
\text { CAT (C/G) GG (T/C) AA (A/C) AGCAGT } \\
\text { GT (G/T) GGCAA (G/A) AC (A/T) TCCTT } \\
\text { TATGC (A/C) GA (C/T) GAC (T/A) CCTT } \\
\text { CAC (G/C) CC (T/A) GCCTT (T/C) GTCA } \\
\text { CAC (T/C) GTGGG (A/C) ATCGACTTTAA } \\
\text { TCG (G/C) (A/C) A (T/A) GA (T/G) AAG } \\
\text { CGT (A/G) T (C/G) AAGCT (C/T) CAGA } \\
\text { T (C/T) TGGGACAC (A/G) GC (G/T) GG } \\
\text { AC (G/C) ACAGCCTATTACCG (G/T) GG } \\
\text { CAT (C/T) CT (C/G) ATGTA (T/C) GACA } \\
\text { ATGAAGA (G/A) TCCTTCAATGCAGTGCA }\end{array}$ \\
\hline XM_001929333 & similar to LOC781710 protein & 0 & $\begin{array}{l}\text { ACAAGCTGGC (T/A) GG (G/A) (A/C) AG } \\
\text { AAC (A/C) A (G/T) GGCTTCC (C/G) TCT } \\
\text { CTC (C/T) ACGGTGC (A/G) (G/A) TCTCA } \\
\text { C (G/C) AAGGGCATCTGGATGTGGTGTGTGCC (T/C) C } \\
\text { ACCCC (A/T) (G/C) (G/C) AATCCAGACCACA (C/T) C } \\
\text { CTGGT (T/C) CT (G/T) CT (T/G) GACACCGAGGGCC } \\
\text { TGGG (G/T) GATGT (A/G) GAGAAGGG (A/C) GA } \\
\text { C (A/T) (A/C) CCAGAA (T/C) GACTC (C/G) TG }\end{array}$ \\
\hline
\end{tabular}


Table S1. Continued

\begin{tabular}{|c|c|c|c|}
\hline & & & $\begin{array}{l}\text { G (C/A) TCTTTGCCCTGGC (A/C) GT (T/G) C } \\
\text { T (C/T) CTGAGCAGC (A/G) CC (C/T) T (T/C) A } \\
\text { T (A/C) TACAACAG (T/C) (G/A) TGGG (G/C) ACC } \\
\text { AGCAGGCC (A/C) TGGA (C/G) CA (A/G) CTGC } \\
\text { ACTA (T/C) GTGAC (A/T) GAACTGACAAAACTCA }\end{array}$ \\
\hline XR_011082 & hypothetical protein LOC702894 & 0 & GAAAAATGATGAGAA (G/A) TCTGCAAAAACAGAT \\
\hline AK240550 & $\begin{array}{l}\text { Sus scrofa mRNA, } \\
\text { clone:UTR010096H04, expressed in } \\
\text { uterus }\end{array}$ & $4 e-147$ & CCGCTCGCTGCCCA (A/G) ACTCCAATCCTGTCG \\
\hline XM_001087923 & $\mathrm{NHL}$ repeat containing 3 & 0 & GTTGG (A/T) CGGGTGTGGGT (G/A) GCTGACC \\
\hline AJ697885 & $\begin{array}{l}\text { cytochrome } \mathrm{P} 450 \text {, family } 2 \text {, subfamily } \\
\text { E, polypeptide } 1\end{array}$ & 0 & C (C/A) AC (C/A) AGCACCACCCTGAGATA (C/T) G \\
\hline XM_001498463 & $\begin{array}{l}\text { similar to lecithin-cholesterol } \\
\text { acyltransferase }\end{array}$ & 0 & TCCATCAAGCCCAT (A/G) CTAGTCTTGGCCTCA \\
\hline BC134783 & carbonic anhydrase VIII & 0 & $\begin{array}{l}\text { TTCTGATCAAGAAC (G/A) GCATACAGCTTCAAAA } \\
\text { GAATGTGAAGGAT (C/T) AGTAAAAATCCTCTCCC }\end{array}$ \\
\hline XM_518045 & similar to C5orf3 protein & $1 e-150$ & $\begin{array}{l}\text { GTTTGATGTTTGCA (G/A) ATATTCTGATTCTCCAG } \\
\text { GTGATGGGGTGGT (A/G) GTGTTTGAATACTGTTG }\end{array}$ \\
\hline NM_183381 & ring finger protein 13 & 0 & CTGCCTGTAGAAGC (A/C) GACGTTTTAGCATATA \\
\hline XM_001109916 & $\begin{array}{l}\text { similar to Zinc finger } \mathrm{CCCH} \text {-type } \\
\text { domain-containing protein } 6\end{array}$ & 0 & $\begin{array}{l}\text { TGGTGCAAATATGTA (C/T) ATAACGTACTGTA } \\
\text { TATTCCGCCCCCTTT (C/T) GTACTCCAGATCT }\end{array}$ \\
\hline $\mathrm{BI} 340566$ & $\begin{array}{l}365876 \text { MARC 2PIG Sus scrofa cDNA } \\
5 ' \text { ', mRNA sequence }\end{array}$ & 0 & $\begin{array}{l}\text { TGGTCACTATAGCCC (A/T) CTAATAAATATTCCA } \\
\text { TGAGATCCTAGCTAA (G/T) CAGAAAACCATTGG } \\
\text { AGCCGAGAAAGAGATTGACA (G/A) TGGTCTTGT } \\
\text { TGTTAC (G/A) TGAG (C/T) GACA (C/T) GATT } \\
\text { AAAATCTG (C/T) GTGTA(T/C) ATTAAATGATATA }\end{array}$ \\
\hline AK234489 & hypothetical protein LOC100157582 & 0 & $\begin{array}{l}\text { CGCCTGCATTGG (G/A) GAGAAGCTAGATGAG } \\
\text { TGTGTGGGCCAT (C/T) GGTACCGGCAAGACT } \\
\text { CCCGCATCATTTA (C/T) GGGGGCTCTGTGACT }\end{array}$ \\
\hline XM_001915087 & hypothetical LOC100057720 & 0 & $\begin{array}{l}\text { T (A/G) TACCC (C/A) (G/A) ACCTC (T/A) C (C/G) C } \\
\text { G (G/A) CT (C/G) T (C/G) (C/G) GACCC (A/C) CA } \\
\text { GGT (C/G) TGG (G/A) T (A/G) GATGC (C/T) G } \\
\text { G (G/C) AC (G/C) CAGATCTT (T/C) TTCTC } \\
\text { T (A/T) CGCCATCTGCCT (G/C) GG (T/G) T } \\
\text { G (T/C) CTGAC (T/G) GC (T/C) CTGGG (A/C) AG } \\
\text { CTA (T/C) AACAA (T/G) TA (T/C) (A/C) ACAACAA } \\
\text { CATC (A/G) (T/C) (G/C) CTCTG (T/C) T (G/T) C } \\
\text { CT (G/C) AA (C/T) AGCGGCACCAGCTTCGTGGCCG } \\
\text { GGTTTGCCATCTTCTC (G/T) (G/A) TCCTGGGCTTC } \\
\text { ATG (G/T) C (G/T) (T/C) A (C/G) GAGCAGGGGG } \\
\text { CAT (T/C) (G/T) C (C/T) GAGGTGGC (A/C) GAGT }\end{array}$ \\
\hline BC112821 & $\begin{array}{l}\text { family with sequence similarity } 107 \text {, } \\
\text { member B }\end{array}$ & 0 & GCACAGAGAACTTCT (T/C) ATGAATCAAAAAAGGGGT \\
\hline BC123755 & RAB8A, member RAS oncogene family & 0 & $\begin{array}{l}\text { AAGTTCATGGAGACC (A/G) GCGCGAAGGCCAAC } \\
\text { TCAAAATCACACCGG (G/A) CCAGCAGAAGAGGA } \\
\text { GTGAGCCCCTCACTC (G/A) GCCGGGGCCCTCC }\end{array}$ \\
\hline XM_001928952 & similar to semaphorin $6 \mathrm{D}$ & 0 & CAATATTAATCCCGG (T/C) GTGCGGAAAATGGA \\
\hline BC134734 & $\mathrm{H} 3$ histone, family $3 \mathrm{~A}$ & 0 & CATGCCAAACGTGTA (G/A) CAATTATGCCAAAAG \\
\hline AK231872 & $\begin{array}{l}\text { similar to Vacuolar proton pump } \\
\text { subunit } \mathrm{E} 1\end{array}$ & 0 & $\begin{array}{l}\text { ATGGA (A/G) TATTACGAGAAGAAGGAAAAG } \\
\text { AACCAGGTACCA (G/A) GTGCTGTTGGATGG }\end{array}$ \\
\hline BC148980 & hypothetical LOC530597 & $9 e-177$ & GCCCAGGGCCTCA (G/A) GTTGGGGCTGGAGGA \\
\hline BC103354 & enolase 1 , (alpha) & 0 & AAGGCTGGCGCTG (C/T) TGAGAAGGGGGTGCC \\
\hline XM_001491791 & $\begin{array}{l}\text { similar to } 26 \mathrm{~S} \text { protease regulatory } \\
\text { subunit } 6 \mathrm{~A}\end{array}$ & 0 & $\begin{array}{l}\text { CAGAGTATGACTCG (A/C) GGGTGAAGGCCATGG } \\
\text { CATTGTCCTGCCAA (C/T) GAACCACAAGGAGAAG } \\
\text { GGGTGCTGATGTAC (G/T) GGCCCCCAGGTACAG }\end{array}$ \\
\hline XM_001928345 & $\begin{array}{l}\text { similar to Acetyl-Coenzyme A } \\
\text { acetyltransferase } 2\end{array}$ & 0 & $\begin{array}{l}\text { CACCGTTCCTGTCC (G/A) TGACCTGGGCTCGAC } \\
\text { TCAGAGAGGTCCTG (G/A) AGAGGGCCAATGTGG } \\
\text { AAAACATGAGCAAG (A/G) CCCCTCACTTGGTTG }\end{array}$ \\
\hline
\end{tabular}


76 Genomics \& Informatics Vol. 7(2) 65-84, June 2009

Table S1. Continued

\begin{tabular}{|c|c|c|c|}
\hline AK233032 & $\begin{array}{l}\text { similar to transmembrane } 4 \text { superfamily } \\
\text { member } 8\end{array}$ & 0 & $\begin{array}{l}\text { CGAGCAGGGCCGC (G/A) GCCGACCGCCGGCGC } \\
\text { CTGTAGGGGCCTTG (T/C) TCTTCGTTATTGGGCTG } \\
\text { CAGCATTCAGAAAGT (G/A) TATAAGACCTACAATG } \\
\text { AAGCTACAAGAAATCA (C/T) GATGCATGTTATCTG }\end{array}$ \\
\hline BC151302 & NDRG family member 3 & 0 & $\begin{array}{l}\text { CTTAAATCTGAAAAGC (G/A) TCATTGGGGTTGGA } \\
\text { ACATTATTTTGGCTCA (C/T) CACTTTGGGCAGGA }\end{array}$ \\
\hline XM_001929069 & pyruvate kinase, muscle & 0 & $\begin{array}{l}\text { ACCTTGAGGCTGAGG (T/C) AGCCGATTCCCGCA } \\
\text { GACTCGCCACCCATC (G/A) CGGCCCGGAACACC } \\
\text { AGTCTGGAATGAACG (C/T) GGCTCGTTTGAACTT } \\
\text { ATGAGTACCATGCAG (G/A) GACCATCAAGAACGT } \\
\text { GGGCAGCGGCACTGC (T/C) GAGGTGGAGCTCAA }\end{array}$ \\
\hline EU282339 & $\begin{array}{l}\text { ATPase, } \mathrm{H}+\text { transporting, lysosomal } \\
13 \mathrm{kDa}, \mathrm{V} 1 \text { subunit } \mathrm{G} 2\end{array}$ & 0 & GAAGTGGAGCAATAC (T/C) GCAGAGAGAGAGAG \\
\hline BX674424 & $\begin{array}{l}\text { BX674424 Sus Scrofa library (scac) Sus } \\
\text { scrofa cDNA clone scac0042.j.05 } \\
\text { 5prim, mRNA sequence }\end{array}$ & 0 & $\begin{array}{l}\text { TGAGAAGACATTCTTC (C/T) CCAAATCTGTTTTCT } \\
\text { GCAGAAGTAGAAGAC }(\mathrm{G} / \mathrm{A}) \text { CACTAGAGTCTGACC }\end{array}$ \\
\hline XM_001925157 & similar to SH3-domain GRB2-like 2 & 0 & GGTCTCAGGTTCTTTA (C/T) TTCAATAGAAGAGAA \\
\hline BC122645 & oligodendrocyte myelin glycoprotein & 0 & АATCTGTCTTATAACCA (T/C) ПTTACTGATCTGCAT \\
\hline BC109483 & $\begin{array}{l}\text { solute carrier family } 4 \text {, sodium } \\
\text { bicarbonate transporter, member } 10\end{array}$ & 0 & AGATGATCCATCTGTG (G/A) TCAATATTTCTGATGA \\
\hline AJ958193 & $\begin{array}{l}\text { AJ958193 KN404_2 Sus scrofa cDNA } \\
\text { clone C0007757o19 5', mRNA } \\
\text { sequence }\end{array}$ & $3 e-145$ & TGATTATTTGGGACCA (G/T) AATTAAGTTAGGTTTT \\
\hline BC118366 & metallothionein $1 \mathrm{E}$ & $8 e-117$ & $\begin{array}{l}\text { CTGCTGTGCCTGATGT (T/C) GGGGAGCACCTGCC } \\
\text { CAACCTGCGTTTTACA (T/C) GACCCTGACTCGAT }\end{array}$ \\
\hline NM_001123139 & myelin oligodendrocyte glycoprotein & 0 & ACCCTGTTTGTGATCG (C/T) GCCAGTTCTCGGACC \\
\hline BC126658 & $\begin{array}{l}\text { phosphotyrosine interaction domain } \\
\text { containing } 1\end{array}$ & $3 e-169$ & $\begin{array}{l}\text { GGAGCCCAATTTATGG (C/T) AACTTATTTACTGGAT } \\
\text { GAATGAGTCCTAGAAA (C/G) CAGACTTATCTAGC } \\
\text { TCTTACAGTTGGGTTG (C/T) TATCTTAGGGTCAG }\end{array}$ \\
\hline XM_532923 & dpy-30 homolog & 0 & ACCTCCAGTCTTTGCC (T/A) ACTCGTGCCTATCT \\
\hline BC103460 & OCIA domain containing 1 & 0 & TCCATTCAGTGCTTCT (G/A) TGAACGAATCTGCTC \\
\hline XM_001928641 & $\begin{array}{l}\text { similar to ubiquitin related modifier } 1 \\
\text { homolog }\end{array}$ & 0 & СCCTTGGCGGTTTTCT (C/T) CCCTAGTCTGTCCC \\
\hline AY566232 & soluble epoxide hydrolase & 0 & $\begin{array}{l}\text { CCGTCGAGTCCGAGG (C/A) GGAGGCCGCCATGG } \\
\text { TCGGGAATACGCCTG (C/T) CTGAGAATTTCTCCGT } \\
\text { GACAAGGCGCTTTCAG (C/A) GAAAAAGATCAACT } \\
\text { ACCCCATGCTGCAGGC (T/C) GCTCTCACTCTCAA }\end{array}$ \\
\hline M29515 & metallothionein & 0 & $\begin{array}{l}\text { GG (G/C) AAGGCGCCTCCTACTGGACCAGTGAATC } \\
\text { G (C/T) TCCTGCAGCTGCGCAGGCTCCTG (C/T) A } \\
\text { TGCTGCTCCTGCTGCCCC (A/G) CGGGCTGTGCA (A/G) GTGTGCCCAGG } \\
\text { (C/G) CTGCATCTGCAAG } \\
\text { AAGTGCAGCTGCTGTGCCTGATGTC (A/G) GGGAG } \\
\text { ACCCTG (T/C) CCCAGAAAT (T/C) AATAGAGCAAC } \\
\text { CTGCATITA (T/A) TATIIT (T/C) AATACAAGCTG } \\
\text { TGAAATATGTGAGTGTAATAAA (ATT) GTTGTCGATTा }\end{array}$ \\
\hline BC118450 & fumarate hydratase & 0 & ATCCAGGCCAACAC (T/C) GAAAGGATCAACAAGCTG \\
\hline BI337595 & $\begin{array}{l}361073 \text { MARC 1PIG Sus scrofa cDNA } \\
5^{\prime}, \text { mRNA sequence }\end{array}$ & 0 & $\begin{array}{l}\text { A (A/G) GACTC (T/C) (T/C) TGGACAT (G/A) G } \\
\text { A (A/G) GT (G/C) AAGCAGAACTTCAT (T/C) G } \\
\text { A (C/T) CCCCT (T/C) CAGAA (T/C) C } \\
\text { T (T/G) (C/T) (A/G) TGACAAGGA (T/C) CT } \\
\text { GA (G/A) GGA (A/G) AT (T/C) CAGCA (T/C) C } \\
\text { A (T/C) CT (A/G) AAGAAG (T/C) TGGA (G/A) G } \\
\text { G (T/C) CG (A/C) CGCCT (A/G) GA (T/C) TTTG } \\
\text { TTA (T/C) AAGAA (A/G) AA (A/G) CG (A/G) C } \\
\text { A (A/G) GGCAAGAT (T/C) CCCGA (T/C) G } \\
\text { A (A/G) GAG (T/C) T (A/G) CGTCA (A/G) G } \\
\text { C (G/C) (C/A) TGGAGAA (A/G) TTTGA (T/G) GA } \\
\text { GTC (T/C) AAAGAAATTGCTGAGTCCAGCATGTTCAA }\end{array}$ \\
\hline
\end{tabular}


Table S1. Continued

\begin{tabular}{|c|c|c|c|}
\hline XM_001498803 & hypothetical protein LOC100053147 & 0 & $\begin{array}{l}\text { ACGGTCATCAGAGGC (C/T) CTCAGGCCGAGCTCAA } \\
\text { GTGCCCCGTGTGTC (C/T) TTTGGAATTTGAGGAGGA } \\
\text { CATTCTGCCCTGGCTGAGCAAG (G/A) CAAACTCCTG } \\
\text { CCCTGTGCCG (T/C) CATGAGCTGCCCACAGATGATG }\end{array}$ \\
\hline U95969 & metallothionein-III & $1 e-179$ & $\begin{array}{l}\text { GCCCTGCCGAGTGTG (G/A) GAAATGCGCCAAGGAT } \\
\text { GTGCCCCTCCGTGC (A/G) AAATGTGTGTAAATAGTGC }\end{array}$ \\
\hline AY864608 & PRA1 family protein-like protein & 0 & $\begin{array}{l}\text { GTGGTGGCTGCCATG (C/A) TGATTTCCGTCGTGGGG } \\
\text { CGGTCTGAAGAGGAC (A/G) CCCATGGGCATTGTCCT }\end{array}$ \\
\hline XM_001926122 & similar to Acylphosphatase- 1 & 0 & $\begin{array}{l}\text { TTATGAAGTTTTTGGA (T/A) AGGTGCAAGGAGTGTTT } \\
\text { CGCAAGTACACTC (G/A) GGCTGAGGGTAAAAAGCTT }\end{array}$ \\
\hline XM_001926433 & $\begin{array}{l}\text { similar to UPF0463 protein C6orf35 } \\
\text { homolog }\end{array}$ & 0 & $\begin{array}{l}\text { GCTGGGACTGCGGA (A/G) TCGTCCTCTCGATTG } \\
\text { TTAGGAGTTCACAGT (A/G) TGAAAGACTTTCGAA }\end{array}$ \\
\hline EU650784 & $\begin{array}{l}\text { cardiac muscle ATP synthase } \mathrm{H}+ \\
\text { transporting mitochondrial } \mathrm{F} 1 \text { complex } \\
\text { alpha subunit } 1\end{array}$ & 0 & $\begin{array}{l}\text { AGTTCCGAGGCTGC (T/G) GCTGCAGAGGAGTCA } \\
\text { CGGC (C/T) GTGGCCCGCGC (T/C) CTCCCTCGG } \\
\text { TTTGTTGCTGCAAGAAA (T/C) CTCCATGCCTCTAA } \\
\text { GACCTTGAAGA (A/G) AC (T/C) GGGCGTGTTTTA } \\
\text { GT (A/G) TACACGGGCTGAGAATGT (C/T) CA }\end{array}$ \\
\hline XM_851307 & SNRPN upstream reading frame & 0 & $\begin{array}{l}\text { TCATGAC (C/T) GTGGG (A/C) AAGAGCAGCA } \\
\text { AGCACAT (T/C) GACTA (T/C) AG (A/G) ATG } \\
\text { AG (A/G) TG (T/C) ATCCTGCAAGA (T/C) GG } \\
\text { CG (G/T) ATCTTCATTGGCACCTTAA (G/A) GC } \\
\text { A (C/T) TTGATCCTCTGTGA (T/C) TGTGA (T/C) G } \\
\text { A (A/G) TTCAG (G/A) AAGATCAA (G/A) CCAAAG } \\
\text { AC (G/T) CAAA (G/A) CA (G/A) (C/G) C (A/G) G } \\
\text { AG (C/A) G (T/A) GAAGA (A/G) AAGCG (G/A) G } \\
\text { T (T/C) (T/C) T (G/T) GGTCTGGTG (T/C) T (G/A) C } \\
\text { T (G/T) CG (T/A) GGGGA (A/G) AA (T/C) (T/C) T } \\
\text { GGT (T/C) TC (C/A) ATGAC (T/G) GT (G/A) G } \\
\text { A (G/A) GGACCACC (C/T) CCCAAAGATACCGG }\end{array}$ \\
\hline DQ499447 & $\begin{array}{l}\text { tRNA-yW synthesizing protein } 3 \\
\text { homolog }\end{array}$ & 0 & $\begin{array}{l}\text { ACCCTAGCTCTCTC (G/T) TCGTATGGATCGGAG } \\
\text { GAACAGTTTTTCAC (C/T) ACCAGTTCCTGCGCT } \\
\text { CCTTGTGTGAAAGA (T/C) GATGTGATTGTAGCC } \\
\text { AGGAAAGACTATGT (T/A) GGCTGTCCGGAGCA }\end{array}$ \\
\hline EW606688 & $\begin{array}{l}\text { rsto213_n2.y1 sto Sus scrofa cDNA } 5^{\prime} \text {, } \\
\text { mRNA sequence }\end{array}$ & $8 e-155$ & ATTCAGTAATTTGT (T/A) CAATATTTTAAAAATT \\
\hline AK236522 & cytochrome b5 type $A$ & 0 & $\begin{array}{l}\text { GAGGTTCGCCGC (G/T) TTACGAAATGGCCGAAC } \\
\text { G (G/A) GAAGAAGT (C/T) (T/C) T (A/G) A (G/T) GG } \\
\text { A (A/G) GCTGG (A/T) (G/A) G (T/A) GATGC (T/A) A } \\
\text { CTGAAA (T/C) TTTGA (G/A) GATGTTGGACA } \\
\text { AC (A/T) GATGC (T/C) (C/A) GAGAG (T/A) T } \\
\text { G (T/C) CCAA (A/G) (A/C) (C/A) GTTCATCAT } \\
\text { TGG (G/T) GA (G/T) (C/G) TGCATCCGAATGA }\end{array}$ \\
\hline XM_001925711 & similar to Protein fam69a & 0 & TGTTGGAAGCTGGAT (T/A) ATCTATGTGCAATA \\
\hline $\mathrm{BC} 105398$ & $\begin{array}{l}\text { CWC15 spliceosome-associated protein } \\
\text { homolog }\end{array}$ & 0 & $\begin{array}{l}\text { GAGAGAGCTGCTGC (G/A) AGAGAAAAAAATAG } \\
\text { TTGTAAATGATACGCT (A/G) CGATCTGAATTTCA } \\
\text { ACAGTTTTATGTGCTT (A/T) ATTAAAGGCTGTAA }\end{array}$ \\
\hline XM_001504320 & $\begin{array}{l}\text { similar to phosphatidylinositol transfer } \\
\text { protein }\end{array}$ & 0 & GTTCAAGTGGTGGGG (T/C) CTGCAGAACAAAG \\
\hline EW310740 & $\begin{array}{l}\text { rpldo0124_e24.y1 Ido Sus scrofa cDNA } \\
\text { 5', mRNA sequence }\end{array}$ & 0 & AGAGGGCTGTCCCC (G/C) СССTCTGATGAGGA \\
\hline CR860378 & $\begin{array}{l}\text { succinate-CoA ligase, ADP-forming, } \\
\text { beta subunit }\end{array}$ & 0 & $\begin{array}{l}\text { CTGCAGACCGCCGC (T/C) CAGGTTCTGGGAAG } \\
\text { ATGAATA (T/C) ATGAGTA (C/T) GGAATTATTG }\end{array}$ \\
\hline AK231804 & $\begin{array}{l}\text { Sus scrofa mRNA, } \\
\text { clone:LNG010025F10, expressed in } \\
\text { lung }\end{array}$ & 0 & $\begin{array}{l}\text { TAGCATCCATGAGGA (C/T) GCGGGTTCCATCCC } \\
\text { CAGAGGGTTAAGG (C/A) TCTGG (T/C) GTTG } \\
\text { TGAGCT (G/T) (T/C) GG (T/C) G (T/A) AG } \\
\text { TC (G/A) CA (G/C) (A/T) (T/C) GTGGCTTG } \\
\text { ATCC (T/C) GCATTGCTG (T/C) G (G/C) CTG } \\
\text { TGG (T/C) (G/A) TAGG (T/C) CAG (T/C) (G/A) G }\end{array}$ \\
\hline
\end{tabular}


78 Genomics \& Informatics Vol. 7(2) 65-84, June 2009

Table S1. Continued

\section{AK293500 Nik related kinase \\ XM_001925969 similar to microtubule-associated protein $1 \mathrm{~A}$}

XM_001929570 heat shock 90kD protein 1, beta AF268463 voltage-dependent anion channel 5, pseudogene

$\begin{array}{ll}\text { XM_001929579 } & \text { similar to SNAP-associated protein } \\ \text { XM_602219 } & \text { collagen, type XXVII, alpha 1 } \\ \text { BC153246 } & \text { prune homolog 2 } \\ \text { AK235825 } & \begin{array}{l}\text { similar to Mitochondrial import inner } \\ \text { membrane translocase subunit Tim23 }\end{array} \\ \text { AK232705 } & \begin{array}{l}\text { Sus scrofa mRNA, } \\ \text { clone:LVRM10010B01, expressed in } \\ \text { liver }\end{array} \\ \text { BC102976 } & \begin{array}{l}\text { NADH dehydrogenase (ubiquinone) 1 } \\ \text { alpha ubcomplex, 11, 14.7kDa }\end{array} \\ \text { AY613916 } & \begin{array}{l}\text { chromogranin B } \\ \text { mitochondrial carrier homolog 2 } \\ \text { NM_014342 }\end{array} \\ \text { XM_001503183 } & \begin{array}{l}\text { similar to CGl-110 protein } \\ \text { thymosin beta 15a }\end{array} \\ \text { NM_021992 } & \end{array}$

XM_001504143 phosphatidylinositol glycan anchor biosynthesis, class $\mathrm{S}$

XM_001929662 similar to MLLT11 protein

XM_001928514 similar to antioxidant protein

BC102595 proteasome (prosome, macropain) 26S subunit, ATPase, 4
CT (A/G) CAGCTCT (T/G)/ ATTG (A/G)/ ACCCCTAG CCTGGGAAC (C/T) TCCATATGCCTCAAGTGTGGCCCTAA

0 CGCATTAAAATTGCT (C/T) TTCGCCTCTGGATCC

0 GTGGTCATCTGCTGTG (G/A) ACCGGGTGTGTAAC GGTTAAAAAGCCACT (G/A)/ CCTGACATCCCAAC ATGTGTGACTCCCCCA (A/G) CATTCCACTATGCCA

0 TGGTGTTTGTGATCC (C/T) TATCCTTTAAGATGCC

0 TGTGA (C/T) CT (T/A) GG (C/A) AA (A/G) GCTG CCA (G/A) (A/G) GAT (A/G) T (T/C) TTCAACAAA GGAT (T/A) TGG (T/G) TTTGG (G/C) (T/A) TGG $T(G / C)$ AAA (C/A) T (G/A) GAT (G/C) TGA (A/G) AA C (A/C) AAGTCATG (C/T) AGTGG (C/A) GT G (A/-) (T/-) (G/-) GAATT(C/T) TC (A/T) AC (A/T) T C (T/G) GGTCATGCT (A/T) A (T/C) AC (A/G) GA (C/T) A C (T/A) GG (T/G) AAAG (T/C) (T/A) (A/T) C (T/A) G $G(G / C)$ A (C/A) C (T/C) TGGAGACCAAATA (T/C) AAGA T (G/C) TGTGA (G/C) TATGG (T/A) CTGAC (T/C) T $T(C / T)$ AC $(A / C)(G / C)$ AAAAGTGGAACAC (T/A) G A (T/C) AA (C/T) ACTCT (G/T) GG (A/G) ACAGAAAT C (G/T) CTTTG (A/G) AGA (C/A) (C/T) A (G/A) (A/G) T

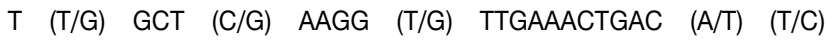
TTGATACCA (C/T) (C/A) TTT (T/G) (C/T) ACCAAA AA (A/G) AGTGG (T/G) AAA (A/T) T (C/G) AAG (T/G) C $(T / C) T C(T / C) T A(C / T) A A(G / A)(A / C) G(G / A) G$ A $(G / A) T G T(G / T) T(A / C)$ A $(A / G)(C / T)(C / A) T T G$ $\mathrm{G}(\mathrm{T} / \mathrm{C})(\mathrm{T} / \mathrm{A}) \mathrm{GT}(\mathrm{G} / \mathrm{A})$ ATGTTGA (C/T) (T/A) T (T/A) GATTTTGCTGGACC (T/A) (G/A) C (T/C) ATC (C/T) ATG $G(T / C) T(C / G)(A / G) G C(T / C) G(C / T)(C / G)(T / C) T$ $(\mathrm{T} / \mathrm{G}) \mathrm{G}(\mathrm{G} / \mathrm{C})(\mathrm{T} / \mathrm{C})(\mathrm{T} / \mathrm{C})(\mathrm{A} / \mathrm{T}) \mathrm{TGA}(\mathrm{G} / \mathrm{A}) \mathrm{GGCTGGC}$ TTGCTGG (G/C) TA (C/T) CAGATGA (C/G) (C/T) TTTGAC A $(G / C)(T / A)$ GCCAAATC (A/C) AA (G/A) CTG (A/T) CA

0 TACACGCCAGGTTATTGGGG (T/C) GCCTGCAGTATCCTCTAC TGGGACTTCCAATTTTCCTTT (G/A) TTTGCACTGAGGATTTAG

3e-149 TTTTTAAGAAAAAAAAAAA (C/A) CCTTATTTAAGATTCTGAAG ACAGACTTTGAAGAAACAT (T/A) TGGACGTGGGGTGTCATCC

2e-165 ACCAAGGGGTCATTGGGG (T/C) AAACGTATTGTTTTCCCAAAG A (C/T) CTTCTAAGACATGCTCTGACCTATGTGGAGAA (G/A) T

0 GTTGAAGCCAAAACCCTC (C/T) GGCAGCTGAGTAATGTGGTTC

1e-160 GCG (T/G) CGTGATGAATGGAAGCTCGCTCGCAGTGATTTTT

\section{9e-169 CATGGCCAAGACGCTTCT (T/C) CACAAGTACTCGGATATCC}

0 TTTCAAATGCCCTGTCGA (A/G) GTCCAATGCTCCTCCCAT

0 ATCCCCTCCTTATTCCCC (G/A) ATATATACTTCTTGGATAG

0 GGCGGGGTCTTTTTGTTG (G/A) TGTCCTATATCTCACTCGGT

5e-160 CTCCCAAGAGTGAATTTCA (A/G) CATTGCTTGAGTGTCTTGA CATCTTCTGATGTCTTCTCA (T/C) CTGTAATGCCTGGCTAAG ACC (T/A) TCAGTGGTGT (A/C) TAGATTCCAGC (C/T) GAC

0 AAGAGTGAAGGGATAATG (G/A) CCTGGGAGCTAGACCGGCT

0 TCCTTTCTTTTCTGGAGGA (C/T) GCCCATTCCAGAACTGGAT

0 AATTCGCCTTTAGCACCA (T/G) TTCTTCCCACCATTCCCCTGCC AAATTATTGCTTTCAGTG (G/A) CAAAGCCAATGAATTTCATGA 0 CCCCTATCCCCACACCC (T/C) AGGAGCTGGGGACTTCTTACCT 
Table S1. Continued

\begin{tabular}{|c|c|c|c|}
\hline XM_001929267 & $\begin{array}{l}\text { similar to ubiquinol-cytochrome } \mathrm{c} \\
\text { reductase binding protein }\end{array}$ & 0 & $\begin{array}{l}\text { AGAACCTTTATAACGAC (G/A) GGGTGTTTCGCATTAAGAGAG } \\
\text { TGGACAAAATATGAGGA (A/G) GATAAATTCTACCTTGAACCA }\end{array}$ \\
\hline XM_001144002 & FGFR1 oncogene partner 2 & $1 e-148$ & CAGTTTTTATTTTTAAACT (T/A) ATTCTATTGATTTGGGGGTAA \\
\hline BC118277 & tetraspanin 7 & 0 & $\begin{array}{l}\text { TTTCATGGAGACCAACA (G/T) GGGGATCATCGCGGGAGTGGC } \\
\text { ACAACGATGGAGTAAGA (G/A) ACCTGTTTCCAAAAGGCATGG }\end{array}$ \\
\hline S73478 & ribonuclease, RNase A family, 4 & 0 & AGCGCTTCAACAC (T/C) TT (C/T) TTCATGAAGACATTTGGAA \\
\hline XM_001928222 & $\begin{array}{l}\text { similar to Myelin-associated } \\
\text { oligodendrocyte } \\
\text { basic protein }\end{array}$ & 0 & $\begin{array}{l}\text { GAAAGTAGCTAAGGAGG (A/G) CCCCAGACTCTCCAAGA } \\
\text { CTGAACGTCAGCC (G/A) CG (A/C) CCCCGCCCAGAAGCCC }\end{array}$ \\
\hline XM_001493074 & similar to DM-20 & 0 & $\begin{array}{l}\text { CAGTA (C/T) GTCATCTA (C/T) GG (C/A) A (T/C) (C/T) G } \\
\text { C (G/C) TC (C/T) TTCTTCTTC (T/C) T (C/T) TA (C/T) G } \\
\text { GG (A/G) (T/C) C (A/C) TCCTG (T/C) TGGC (A/T) GAGG } \\
\text { GCTTCTACACCACC (A/G) GCGCAGTCAGGCAGATCTT }\end{array}$ \\
\hline XM_001497146 & $\begin{array}{l}\text { adaptor-related protein complex } 2, \mathrm{mu} \\
1 \text { subunit }\end{array}$ & 0 & $\begin{array}{l}\text { CAGG (C/T) GAGCAGGGGGCGGGCGGACA (T/C) CTTG } \\
\text { TITGTGCTCATATATGAG (C/T) TGCTTGATGAGATCCTGGAC } \\
\text { GGCAGATTGGCTGGCGG (G/C) GGGAAGGCATCAAGTATCG }\end{array}$ \\
\hline XM_001103332 & sepiapterin reductase & 0 & CTCCCGCGGTTTCGGAC (T/G) GACGCTGGCACAGCTCCTG \\
\hline CR860856 & $\begin{array}{l}\text { NADH dehydrogenase (ubiquinone) } 1 \\
\text { alpha subcomplex,1, } 7.5 \mathrm{kDa}\end{array}$ & $2 e-126$ & AGTGTATTTTGTAGTATGTAATAAAAA (C/T) TTTAAAAGT \\
\hline $\mathrm{BI} 346028$ & $\begin{array}{l}\text { 375023 MARC 2PIG Sus scrofa cDNA } \\
5 \text { ', mRNA sequence }\end{array}$ & 0 & TGCGATCACAACTGAAC (G/A) TAGATAGCAAAACACTTA \\
\hline NM_001113234 & $\begin{array}{l}\text { anaphase promoting complex subunit } \\
13\end{array}$ & $3 e-168$ & ACATTGGCTTATTTTGT (G/A) GCAGCAAAATGCAATGTG \\
\hline AW787203 & $\begin{array}{l}120964 \text { MARC 1PIG Sus scrofa cDNA } \\
5 \text { ', mRNA sequence }\end{array}$ & 0 & TCATTGACCAATTGACAA (T/C) GTGGTTTAACTATCAAGA \\
\hline XM_853711 & $\mathrm{H} 3$ histone, family $3 \mathrm{~B}(\mathrm{H} 3.3 \mathrm{~B})$ & 0 & $\begin{array}{l}\text { CGTTCGTTGGAGTTGCT (A/T) CTTGCTTTGGTTCGGCGA } \\
\text { TTGGTGAGGGAGATCG (T/C) CCAGGATTTCAAAACTGAC }\end{array}$ \\
\hline BC102690 & $\begin{array}{l}\text { NADH dehydrogenase (ubiquinone) } 1 \\
\text { beta subcomplex, } 5,16 \mathrm{kDa}\end{array}$ & 0 & CGTGGTATCAGTATCC (G/A) ACCATTGATAAGGCACTTAT \\
\hline BC150010 & SEC11 homolog A & 0 & GATTGTGACGATCCTC (G/A) TGAATGACTATCCTAAATTTA \\
\hline AK161097 & $\begin{array}{l}\text { FUS interacting protein (serine-arginine } \\
\text { rich) } 1\end{array}$ & 0 & AATACGTCTCTGTTCGT (A/G) AGGAACGTGGCCGACGATA \\
\hline BC102721 & $\mathrm{NAD}(\mathrm{P}) \mathrm{H}$ dehydrogenase, quinone 2 & 0 & $\begin{array}{l}\text { AAACCTCGCGCAGCCTT (C/T) CCGCTGCGCCCCTCAGGCG } \\
\text { GCGCCCTCTCCAACCCC (A/G) GCTTCTTCAATTACGGGGTG }\end{array}$ \\
\hline CJ025998 & $\begin{array}{l}\text { CJ025998 full-length enriched swine } \\
\text { cDNA library, adult trachea Sus scrofa } \\
\text { cDNA clone TCH01B050048 5', mRNA } \\
\text { sequence }\end{array}$ & 0 & $\begin{array}{l}\text { TACAC (T/A) TCTGTCTGCTGGTATTAGCAGC (T/C) TGAG } \\
\text { TGAGCCTCTGTGTTCCAA (G/A) GGCTGCGTGCTCTGCTTGA }\end{array}$ \\
\hline BC102779 & septin 6 & 0 & $\begin{array}{l}\text { GCT (G/C) CGG (C/T) CC (C/A) A (G/T) ACCTA (C/T) GAC } \\
\text { T (C/T) CAAGAGAGCAACGTG (C/G) (A/G) (G/C) CT (C/G) A } \\
\text { CT (G/C) ACCATCGT (G/T) (G/A) (A/G) C (G/A) C (C/A) GT } \\
\text { (T/A) GA (G/C) AG (T/C) TACA (G/A) GCCCATCGT (C/G) G } \\
\text { A (C/A) T (A/T) CATCGACGC (G/C) CA (G/A) TTTGA (A/G) (A/G) } \\
\text { (A/C) CTA (C/T) CTGCAGGAGGAGCTGAAGATCCG } \\
\text { C (C/A) G (C/G) (T/G) (C/T) (G/A) CT (C/G) (T/C) (T/A) C } \\
\text { (G/A) (A/C) CTACCACGAC (A/T) C (G/C) (A/C) G (G/C) AT } \\
\text { CCACG (T/C) CTGC (C/T) T (C/G) TACTTCATCACGCCCACCG }\end{array}$ \\
\hline XM_001500749 & hypothetical protein LOC100064910 & 0 & $\begin{array}{l}\text { TCTGGCTCTGCGAGTACT (G/A) TCTCAAGTACATGAAATATGA } \\
\text { GGAGCC (G/A) TTCGTCTTCTACATCTTGAC (T/C) GAGGTGGAC } \\
\text { AAGCCGCTGTCTGACCTG (G/A) GCAAGCTCAGCTACCGCAGC }\end{array}$ \\
\hline BC148053 & solute carrier family 25 , member 38 & 0 & $\begin{array}{l}\text { CCСCTGACTGGCGCAGAC (C/T) ACAAAGAGGCCCCCGCAGTA } \\
\text { CGGCAACCCGGGCCCAGT (A/G) CACTCGCAGGCCTCCCCTCC } \\
\text { AGCCCATGGATCCAGACGC (A/G) TTGGGATGTTGGCTTTGCTC } \\
\text { AGAGTCTGCTGGGCCTTTG (G/A) AAAGGGATGTCTCCTTCCAT }\end{array}$ \\
\hline AK236031 & similar to MRPL43 protein & 0 & $\begin{array}{l}\text { GCCCTCTTCCCGCGGTGCC (G/A) GGGAGTTTGTGGAACGGGA } \\
\text { AGTTGCCCCATTAACTCCA (G/A) TTCCAGTCTTGATTCTCATC } \\
\text { GGTGTTCACTGTGAGGGGC (C/A) GTGGCTGTGAGCCTTCTAGT }\end{array}$ \\
\hline
\end{tabular}


80 Genomics \& Informatics Vol. 7(2) 65-84, June 2009

Table S1. Continued

BC126591 FYN oncogene related to SRC, FGR,
YES

D37917

\author{
cathepsin L
}

XM_525719

XM_001498796

abhydrolase domain containing 1

similar to phosphatidylinositol glycan,

class $F$

D90073

NM_001040457

BC102096

poly (ADP-ribose) polymerase 1

rhomboid domain containing 2

eukaryotic translation initiation factor 3 , subunit $\mathrm{H}$

AY911314

AY166682

splicing factor $3 \mathrm{~b}$, subunit $5,10 \mathrm{kDa}$ transmembrane BAX inhibitor motif containing 6

XM_001928357 similar to RAB25
0 ATGATCCACTGCTGG (A/C) (A/C) AAAGGACCC (C/G) GAAG A (G/A) (A/C) GGCCCAC (T/C) TTCGAGTAC (T/C) TGCAGG (G/C) CTTCCTGGAAG (C/A) CTACTTCACCGCGAC (A/C) GA CCCAGTA (T/C) CA (A/G) CCCGG (T/A) GA (A/G) AACCTGT ATCCTGTTG (C/G) TAT (T/A) GATGC (G/A) (G/A) GCCA T (T/G) (C/A) ATC (T/C) TTCCAGTT (C/T) TA (T/C) A (A/G) (G/C) (T/A) CAGGCATTTATT (A/T) TGA (T/A) CC A $(G / A)$ A (C/G) TGCAGCAGC (A/G) AAGA (C/T) CTGGA G (T/C) GTT (T/C) TGGTGGT (T/C) GGCTATGGC (T/G) T GGA (A/G) C (T/A) (G/C) A (T/C) TCAAATAATAA (A/G) T (T/A) TTGG (A/C) TTGTCAAGAACAG (T/C) TGGGGTAATAC

0 TTCCAGGGGAGCAGCCC (C/T) TCCAGGAAGCCGATGTGGCC

0 CTTCTCAATATTGGAAAC (G/A) CACTTGACATGGTTGTGCATC AGTAGTGAAATCAAATAC (A/G) TCATCTAAAAGAAGTTCATT

0 TCACTG (G/A) ATCTCCTTAGAAAGGATTTACATGAACA

0 CCGTCCGCGCTCGGATG (A/C) GGCGGGCCCTGGTGTTTGGC

0 CAGCTCCACCACCGGC (G/A) CTGCTGGGAAAGGCAAAGGC ACTTCTGGATTCTCAGTT (C/T) AGTTACCAGCATGCGATTGAA ATAAAAACTGCCCAAGG (G/A) TCTCTCTCACTAAAGGCATAC

5e-160 CTTCTTCTCGTCTGCGGC (C/T) TCTGACCTGCGAGCAAAGGA

0 AATTCTCTGCTAGGCCTG (G/A) GAATCTGAGTTACATCTCTTG

0 TttgtcttcaA (A/G) GTGGTGCT (C/G) ATCGG (G/C) G A (T/G) TC $(G / A)$ GG $(A / C)$ GTGGGGAAGA $(G / C)$ CA A (C/T) CTGCT (G/A) TCCCG (C/A) TTCAC (C/T) CG AA (C/T) GAGTTCA (A/G) CC (T/A) (G/C) GA (G/C) A $\mathrm{GC}(\mathrm{A} / \mathrm{C})(\mathrm{A} / \mathrm{G})(\mathrm{G} / \mathrm{C}) \mathrm{A}(\mathrm{G} / \mathrm{C})$ CACCATCGG $(\mathrm{C} / \mathrm{G}) \mathrm{G}$ T (G/T) GAGTTT (G/T) CCACCCGCA (G/C) (C/G) (A/G) T C (C/A) (A/T) G (G/C) TGG (A/G) C (G/A) (G/C) C $(\mathrm{A} / \mathrm{G})(\mathrm{A} / \mathrm{C})(\mathrm{G} / \mathrm{C})(\mathrm{A} / \mathrm{G}) \mathrm{C}(\mathrm{C} / \mathrm{T})(\mathrm{A} / \mathrm{G})$ TCAAGGC $(\mathrm{A} / \mathrm{T}) \mathrm{C}$ AGATCTGGGACAC (C/G) GC (C/T) GGCC (A/T) GGAG CG (C/A) TACCG (G/A) GCCATCACCTC (T/G) GC (A/G) TA TA (C/T) CGTGGTGCAGTGGG (T/G) GC (G/C) CT (G/A) CT GGTGT (A/T) TGA (T/C) (A/C) T (T/A) (G/A) CCAAGCAC C (T/A) GACATACGAGAACGTGGAGCGCTGGCTGAAG

0 GGCCTGGAAGCAAATGTC (G/C) TGGTTCTACTACCAGTACCT

0 GTCGACCCACGCG (G/T) CC (C/G) CTCGGCGGTCGGAAGG GGCATC (T/A) CCTCGGTCCGGGCTACTGCCA (T/A) CATGC AAGCCCAACAAGGAGTTCA (C/T) GCTCTGCCGGTGGGAAGA AAGAAC (C/T) TGACATAGT (G/A) TCCA (C/T) (A/G) (G/A) G GA (A/T) GC (C/A) GGTT (C/T) (A/G) ATCCCT (G/T) G $(\mathrm{C} / \mathrm{T})(\mathrm{A} / \mathrm{C}) \mathrm{T}(\mathrm{C} / \mathrm{T})(\mathrm{A} / \mathrm{G}) \mathrm{CTCAG}(\mathrm{T} / \mathrm{G}) \mathrm{GG}(\mathrm{A} / \mathrm{G})$ TTAAGGA

6e-101 AACTTGGAGTTC (T/C) CTTGTGG (T/C) (G/A) CA (A/G) C AGGTT (A/G) AGGAT (T/C) (T/C) (G/A) GCATTGTC (A/C) C TGC (C/T) (A/G) TG (A/G) (C/T) G (A/C) (G/A) (A/G) G TT (T/C) (G/A) ATCCTTGGC (C/T) (C/T) AGAAACTTCCACA

0 AAGCCA (G/A) GCTCCGCCTCTTGGGCAG (T/C) TTTGACT

0 CACCGAGAGAGCGCTGAC (A/G) GCCCTGCTGAAGTGTGCA

0 ACCCTCGGTGCAGCTGGT (G/A) CAGAGCTGGTATGTCCAGA ACCCTGAGCCAGTTCACC (A/G) ACGCCCTGGTCACCATCCG - GAAGGACAAGGTTCGGCG (C/T) GCGCTTGAAGAGAACCGC GAGTTACGCAGAGAGGCT (T/C) TGGCCTTACAGGGATCCCT CGGCACGAGGTCGGGGC (A/G) CTGGTGCGAGCCTGCAAAG 0 ATTAAATCTCAACTAATGAA (C/T) CTGATTCGATCTGTAAGAA CAGCAGAAGTTACTACTTCA (A/G) TCTTTGTTGGAATATACAT 3e-30 GCTGAAATCCCAGCTG (A/G) (A/G) TGTCTCTTTCCCTAAA 
Table S1. Continued

\begin{tabular}{|c|c|c|c|}
\hline EF416570 & P311 protein & 0 & $\begin{array}{l}\text { TGAATGTTGTTCTCTAGAC (C/T) GGATTGACTTGTTTTCCTTG } \\
\text { AGTAAGTGCCACCTCTGAG (G/A) GCAGACACTCGTCTCAGG } \\
\text { TTGGGGAAAAAAAGGGCAA (C/A) CTGGTTTGGGTTTTTTTGT }\end{array}$ \\
\hline XM_001929537 & ubiquitin-conjugating enzyme E2L 3 & 0 & CACGTTTAAAACGAAGATCT (G/A) TCACCCCAACATCGATGA \\
\hline XM_001168089 & contactin 1 & 0 & AACTAAATCTTTTGCCAAAT (G/A) CATGCTTGCCTTTTATTTT \\
\hline XM_001503420 & similar to visinin-like protein & 0 & AGAGCGGCCCTTGGCTCCT (T/C) GGTGTGGTCCCCACACAA \\
\hline XM_533961 & cold inducible RNA binding protein & 0 & $\begin{array}{l}\text { ACTCAGTGGTGACCATGG (C/G) ATCAGACGAGGGCAAACTTT } \\
\text { ATGGCCATGAACGGGAAG (C/T) CTGTGGATGGGCGGCAGAT } \\
\text { GCCGGAGTCAGGGTGGC (A/G) GCTATGGTGACCGGAGCTCA }\end{array}$ \\
\hline XM_001927751 & $\begin{array}{l}\text { similar to kidney predominant protein } \\
\text { NCU-G1 }\end{array}$ & 0 & CCTGCCACCCTGAGTGCC (G/A) CATTTCGAGGCCACCCCATT \\
\hline XM_001495383 & similar to Protein ARMET precursor & 0 & GACTGATTTGTAGTCTGCC (G/C) AATCCCTGCTGCACCTGAGG \\
\hline XM_614056 & cullin 3 & 0 & TTAAATATTAAGAAGACC (G/A) TCTCTTCTATTAAATTACAGT \\
\hline NM_001791 & cell division cycle 42 & 0 & $\begin{array}{l}\text { A (G/A) AGG (T/C) CT (G/A) AAGAATGT (G/A) TTT (A/G) A (T/C) GA } \\
(\mathrm{G} / \mathrm{A}) \mathrm{GC}(\mathrm{T} / \mathrm{A}) \text { AT (C/A) (C/T) T (A/G) GC } \\
\text { TGCCCT (C/G) GAGCCTCC (G/A) GAACCGAAGA (G/A) GA }\end{array}$ \\
\hline AK233807 & ribosomal protein $\mathrm{S} 4$ & 0 & $\begin{array}{l}\text { CCGGTGTGTTTGCTCCTCG (C/T) CCATCTACTGGTCCCCACAA } \\
\text { GCTGTTCATCGTATTACACC (G/T) GAGGAGGCCAAGTACAAG } \\
\text { CTAACCTGGGAAGAATTGG (C/T) GTGATCACCAACAGGGAGA }\end{array}$ \\
\hline AK234517 & glucosidase, beta; acid & 0 & GGCCATGACAGATGCTGC (C/T) GCTCTCAACATCCTTGCCCT \\
\hline XM_001925668 & similar to Neurocalcin-delta & 0 & TATAAG (G/A) TGGTTTCCTCTGTGATGAAAA (C/T) GCCTGA \\
\hline BC126729 & $\begin{array}{l}\text { heat shock protein, } \\
\text { alpha-crystallin-related, B6 }\end{array}$ & 0 & $\begin{array}{l}\text { GAGGAGCG (C/G) C (C/A) GGATGA (G/C) CACGG (A/C) TACA } \\
\text { TC (G/T) C (G/C) CGCGAGTTCCACCGCCGCTACCGC (T/C) TG } \\
\text { CCGCCTGGTGTC (A/G) ACCCGGCGGCCGTGACGTCCGCTCTG }\end{array}$ \\
\hline U73504 & $\begin{array}{l}\text { calcium/calmodulin-dependent protein } \\
\text { kinase II delta }\end{array}$ & 0 & $\begin{array}{l}\text { CCTGCACC (A/C) G (G/C) TTCAC (G/C) GACGAGTA (T/C) C } \\
\text { AGCT (T/C) T (T/A) CGAGGAG (C/A) TTGG (A/C) AAGGGGG } \\
\text { C (A/T) TTCTC (A/T) GTGGTG (A/C) GAA G (A/C) TGTATGA } \\
\text { A (A/G) (A/C) TC (C/T) C (T/C) AC (T/C) GG (A/C) CA (A/C) G } \\
\text { A (A/G) TA (T/C) GC (T/A) GCCAA (A/G) ATTATCAACACCAA (A/G) } \\
\text { AAGCT (T/C) TCTGC (T/C) AG (G/A) GATCATCA (A/G) } \\
\text { A (A/G) CTGGA (A/G) AGGGA (A/G) GCT (A/C) GAATCTGCCG } \\
\text { CTT (T/C) TGAAGCAC (C/T) CAA (T/C) AT (T/C) GTGCGACT } \\
\text { CTT (T/C) CATTACTTGGT (G/C) TT (T/C) GA (T/C) (T/C) TGG } \\
\text { T (T/C) ACTGG (A/C) GG (T/G) GA (A/G) CT (G/C) TTTGAA } \\
\text { GACAT (A/C) GTGGCGAGAGATATTACAG (T/C) GA (A/G) G } \\
\text { C (T/G) GA (T/C) GCCAGTCA (T/C) TGTATACAGCAGATTCTA } \\
\text { GACCAACACCGACTCTA (C/T) CAGCAGATCAAGGCTGGAGCT }\end{array}$ \\
\hline BC122616 & profilin 2 & 0 & $\begin{array}{l}\text { TGGCCGGTTGGCAGAGC (C/T) ACGTGGATAACCTGATGTGCG } \\
\text { CTGCCAGGAAGCCGCCA (C/T) TGTCGGCTACTGCGACGCCAA } \\
\text { GTGGGGAGCCAACATAC (G/A) ACGTTGCTGTCGGCAGAGCTG }\end{array}$ \\
\hline AK236785 & $\begin{array}{l}\text { Sus scrofa mRNA, } \\
\text { clone:PBL010004E06, expressed in } \\
\text { periphral blood mononuclear cell }\end{array}$ & 0 & GTGCCCATCAGACCTCG (C/T) TCAAACCCTCATGCATTTCGAG \\
\hline BC134490 & SUB1 homolog & 0 & CAAGTTGCTCCAGAAAA (G/A) CCTGTAAAGAAGCAAAAGAC \\
\hline XM_857035 & $\begin{array}{l}\text { heterogeneous nuclear } \\
\text { ribonucleoprotein } \mathrm{A} 3\end{array}$ & 0 & TGGACACTGTCAATGTT (T/C) ATAACTTAAACCTGGGTACAT \\
\hline XM_001928960 & $\begin{array}{l}\text { similar to Astrocytic phosphoprotein } \\
\text { PEA-15 }\end{array}$ & 0 & GCCATGTCTGCCCTTTT (T/A) TTGTGGTGTCTAGCATTAACTT \\
\hline AK238034 & poly $(\mathrm{rC})$ binding protein 2 & 0 & $\begin{array}{l}\text { AAGCTGCACCAG (T/G) TGGCAA (T/G) (G/A) CAACAGTCTCAT } \\
\text { CCCATGACGCA (T/C) GGCAACACCGGATTCAG (T/C) GGCATT } \\
\text { (A/C) TCCAGCTCTCCAGAGGTGAAAGGCTATTGGGCA (G/A) G } \\
\text { TTGGATGCATCT (G/A) CTCA (G/A) AC (T/C) ACTTCTCATGAA } \\
\text { AACGA (T/C) TTGATTGGCTGCATAATCGGGCG (T/C) CAAGGC } \\
\text { CAA (A/C) AT (C/T) AATGAGATCCG (T/C) CAGATGTC (T/C) G } \\
\text { AAAATTGC (G/C) AA (C/T) CCAGTGGAAGG (A/C) TCT (A/T) C } \\
\text { (A/G) TAGGCAGGTTACCATCACTGGATCTGCTGCCAG (C/T) A } \\
\text { TAG (C/T) CTGGC (T/C) CAATATCTAATCAATG (T/C) CAGGCT }\end{array}$ \\
\hline
\end{tabular}


82 Genomics \& Informatics Vol. 7(2) 65-84, June 2009

Table S1. Continued

\begin{tabular}{|c|c|c|c|}
\hline BC153235 & chimerin (chimaerin) 1 & 0 & $\begin{array}{l}\text { GAAGA (C/T) AT (A/C) AACAT (C/T) ATCACTGG (A/T) G } \\
\text { C (C/G) CT (T/G) AAACTGTACTTCAG (A/G) GA (C/T) T } \\
\text { TACC (C/A) ATTCC (T/A) (G/C) TCATCAC (C/G) TATG } \\
\text { A (T/C) (A/G) CCTA (T/C) (T/C) C (T/G) AA (A/G) T } \\
\text { T (T/C) ATAGA (A/G) (G/T) C (A/T) GC (A/C) AAAATC }\end{array}$ \\
\hline AY609871 & $\begin{array}{l}\text { Sus scrofa clone } \\
\text { Clu_36963.scr.msk.p1.Contig1, mRNA } \\
\text { sequence }\end{array}$ & 0 & TAAGACAGCTATGTCTCA (A/G) CCTGACTCTTGACATTAA \\
\hline BQ597566 & $\begin{array}{l}\text { MI-P-A2-afc-a-12-1-UM.s1 MI-P-A2 Sus } \\
\text { scrofa cDNA clone } \\
\text { MI-P-A2-afc-a-12-1-UM 3', mRNA } \\
\text { sequence }\end{array}$ & 0 & $\begin{array}{l}\text { GGAGTTCCC (G/A) TCGTGGCT (C/A) AGTGG (T/A) TAAT } \\
\text { GAA (C/T) (A/C) (C/T) GACTGGCATCCATGAGGACACAGG } \\
\text { CCTGGC (C/A) TC (G/A) CT (C/T) AGTGGGTTAA (G/A) G } \\
\text { ATCTGG (T/C) GTT (G/A) C (C/A) GTGA (G/A) CTGTGGT } \\
\text { GTAGG (T/G) TG (T/C) AGATG (C/T) GGCTCA (G/A) ACC } \\
\text { GC (G/A) T (T/C) G (T/C) TGTG (C/G) CTGTGG (C/T) G } \\
\text { CCAGCGG (T/C) T (A/G) CAGCTCCAATTCA (A/G) CCCCT } \\
\text { GCCTGAGAAACTCCATATGC (T/C) GC (T/A) GGTGCAGCC }\end{array}$ \\
\hline XM_001925602 & similar to claudin 10 & 0 & $\begin{array}{l}\text { TCTCCATCTCCGGCTGGGTG (C/T) TGGTGTCCTCCACGCT } \\
\text { GCCCACCGACTACTGGAA (G/A) GTGTCCACCATCGACGGC }\end{array}$ \\
\hline BE013772 & $\begin{array}{l}125231 \text { MARC 1PIG Sus scrofa cDNA } \\
5^{\prime}, \text { mRNA sequence }\end{array}$ & $4 e-179$ & $\begin{array}{l}\text { TTTTTATCAG (G/A) GTCTTCACGG } \\
\text { TTGTAGGG (G/A) AGACGTTGTACT } \\
\text { CTTTCTTTTC (G/A) ATTGTGGCTCT } \\
\text { GTCGCGGA (T/-) (T/-) TGTTAGTGTAG } \\
\text { TAACTTCTCAAATC (T/C) CTT } \\
\text { GCTTCCTTTT (T/A) GTCTCAATGG } \\
\text { GTCCAGGTT (T/C) TGAATCCACTCA }\end{array}$ \\
\hline CN157594 & $\begin{array}{l}946310 \text { MARC 4PIG Sus scrofa cDNA } \\
\text { 5', mRNA sequence }\end{array}$ & 0 & TCСTCTT (C/T) CTGTGATGATTCCATCTGTTT \\
\hline EW089596 & $\begin{array}{l}\text { recc2802c_h7.y1 ecc Sus scrofa cDNA } \\
5 ', \text { mRNA sequence }\end{array}$ & 0 & AGATAAATCACAGA (G/A) CGTGCCGTT \\
\hline AW347194 & $\begin{array}{l}30566 \text { MARC 1PIG Sus scrofa cDNA } \\
5^{\prime}, \text { mRNA sequence }\end{array}$ & $5 e-134$ & $\begin{array}{l}\text { CCGCCTCTTC (T/C) ACTCTTGGTGAC } \\
\text { GTTTTGTGTCT (C/T) GATCCCCGTGG } \\
\text { AAGAGCCCGG (T/C) GCACACACAGC }\end{array}$ \\
\hline AK235753 & $\begin{array}{l}\text { Sus scrofa mRNA, } \\
\text { clone:OVRM10111F12, expressed in } \\
\text { ovary }\end{array}$ & 0 & CCAGCTGTAGA (G/A) AAGAATATATC \\
\hline EV903443 & $\begin{array}{l}\text { rcbl0_004449.y1 cbl Sus scrofa cDNA } \\
\text { 5', mRNA sequence }\end{array}$ & 0 & AAT (A/G) ATCAAATGCCACTGTCA (T/C) TGC \\
\hline CN158340 & $\begin{array}{l}947125 \text { MARC 4PIG Sus scrofa cDNA } \\
\text { 5', mRNA sequence }\end{array}$ & 0 & TCATTGGAAACCT (T/C) GTCTAACAACAGAAAC \\
\hline BG895945 & $\begin{array}{l}359644 \text { MARC 1PIG Sus scrofa cDNA } \\
\text { 5', mRNA sequence }\end{array}$ & $1 e-179$ & $\begin{array}{l}\text { GCT (G/A) TGGC (T/C) GTGG (T/C) A (T/C) AGG } \\
\text { CTC (T/C) GATTCAAC (A/C) (A/C) C (T/A) AGCC } \\
\text { CCTGGGAGTTTC (T/C) ATATGCCACAGGTACAACCC }\end{array}$ \\
\hline BW982861 & $\begin{array}{l}\text { BW982861 full-length enriched swine } \\
\text { cDNA library, adult intestine Sus } \\
\text { scrofa cDNA clone ITT010077F03 5', } \\
\text { mRNA sequence }\end{array}$ & 0 & $\begin{array}{l}\text { AGGA (T/G) CCTCCTCAACTCTGTCT (G/A) (G/T) GA } \\
\text { TG (C/T) TGGGTCAGC (A/G) TGTTCGCCA (A/G) TTT } \\
\text { GGTGAGAA (G/A) CTGGTCCGAAGGCGGCCACTGGAG } \\
\text { CCCAG (C/G) GACGAGTCTGAGATTCCCACACCTCATC } \\
\text { T (A/C) AACA (C/G) C (C/T) T (C/T) (A/G) A (T/C) C } \\
\text { TG (C/G) T (T/C) TTTGGTGTGAA (T/C) A (T/G) AAC } \\
\text { CT (A/T) AGG (C/G) T (C/T) GGT (A/G) T (A/G) TA } \\
\text { CTGGT (G/T) C (C/A) (A/G) TGGCCGTG (C/T) ATG } \\
\text { TAGCTGGACCTG (C/G) (T/G) ATCATC (A/G) TCTC } \\
\text { C (T/C) TCTTG (C/G) T (C/G) G (T/C) (T/C) G (G/C) C } \\
\text { TT (A/C) T (T/C) T (G/T) C (C/T) (G/A) TGTT } \\
\text { G (T/G) (G/C) TG (C/G) (A/G) (A/T) T (C/G) T (G/A) C } \\
\text { T (T/A) (T/C) GC (T/A) GAGTTTGG (G/T) GCCCGGG } \\
\text { T (A/C) (T/C) CC (T/C) G (C/T) T (G/C) (T/A) GG } \\
\text { G (T/C) GTATCTCTACAGCTATGT (G/C) AC (A/T) (A/G) T } \\
\text { GGG (A/T) (C/G) AACT (G/C) T (A/G) (T/G) GCCTTC } \\
\text { A (T/C) CACTGGCTGGACCCTCATACTGCATTTACGCGT }\end{array}$ \\
\hline
\end{tabular}


Table S1. Continued

\begin{tabular}{|c|c|c|c|}
\hline BF191147 & $\begin{array}{l}237886 \text { MARC 2PIG Sus scrofa cDNA } \\
5 ' \text { ', mRNA sequence }\end{array}$ & 0 & СCTATATTCCATTC (G/A) TAGTTGAACCATGCAGTACC \\
\hline DR066496 & $\begin{array}{l}\text { rp11895 Normalized porcine adipose } \\
\text { tissues cDNA library Sus scrofa cDNA, } \\
\text { mRNA sequence }\end{array}$ & $4 e-170$ & $\begin{array}{l}\text { C (T/A) TCCAGTGTTGCTGTGGCTGTGGC (A/G) TAGG (T/C) C } \\
\text { AGCA (C/G) CTGCAGCTCC (G/A) A (G/T) T (T/C) G } \\
\text { A (T/C) CCCT (G/A) GCC (C/T) GGGACTTCCATATGCTGC }\end{array}$ \\
\hline XM_001493250 & $\begin{array}{l}\text { kelch repeat and BTB (POZ) domain } \\
\text { containing } 7\end{array}$ & 0 & $\begin{array}{l}\text { CT }(\mathrm{G} / \mathrm{T}) \text { TA }(\mathrm{C} / \mathrm{T}) \mathrm{GC}(\mathrm{C} / \mathrm{T})(\mathrm{C} / \mathrm{G}) \mathrm{T}(\mathrm{C} / \mathrm{G}) \text { AA } \\
\text { GCAAGCGCATG (T/C) TCTGCT (T/A) (T/C) GAT } \\
\text { CCCAGCCA (C/T) AACATGTGGCTGAA (G/C) TG } \\
\text { TGC (G/T) TCTCTTAAACGCAGTGACTTCCAGGAG }\end{array}$ \\
\hline XM_001929045 & hypothetical LOC100155420 & 0 & AAATCTAGTTTTTGA (T/C) TTGTTTTTGTCTTGTT \\
\hline XM_585907 & carbonic anhydrase XIV & $1 e-162$ & $\begin{array}{l}\text { GTCTGTGTGAATAGC (A/G) AAGGAAGGAAGAGC } \\
\text { TCGCCAGCAACCCAG }(\mathrm{G} / \mathrm{T}) \text { CAACCCCCCCTCCC }\end{array}$ \\
\hline EW646381 & $\begin{array}{l}\text { rthg04_l18.y1 thg Sus scrofa cDNA 5', } \\
\text { mRNA sequence }\end{array}$ & 0 & ACAATCATTTTTTTTTC (T/C) TTCATTAGCCATTAT \\
\hline BE234333 & $\begin{array}{l}141171 \text { MARC } 1 \text { PIG Sus scrofa cDNA } \\
5 ', \text { mRNA sequence }\end{array}$ & 0 & $\begin{array}{l}\text { CCACA (G/A) AACATCTACTG (T/A) ACACTGGCA } \\
\text { GA (A/T) C (T/C) TAA (G/A) (C/T) CTC (A/C) AA }\end{array}$ \\
\hline BQ597486 & $\begin{array}{l}\text { MI-P-A2-aey-h-04-1-UM.s1 MI-P-A2 } \\
\text { Sus scrofa cDNA clone } \\
\text { MI-P-A2-aey-h-04-1-UM 3', mRNA } \\
\text { sequence }\end{array}$ & 0 & $\begin{array}{l}\text { CACACAGCATGAA (G/C) TGATCAAGAAAAGCCACA } \\
\text { CACAACCTGTTTA (G/C) ACTGATGAAGCTTCTAGGA } \\
\text { GAACTGTGTC (A/G) TGAAACCAGTGAGGAAGAAG }\end{array}$ \\
\hline BC120117 & NDRG family member 4 & 0 & $\begin{array}{l}\text { AGGG (G/A) AACCG }(C / G) \text { CC }(A / C) \text { G }(C / T) \text { CATCC } \\
\text { TGAC }(G / A) T(G / T) G G(T / C)(C / A) T(C / G) \text { AACC } \\
\text { ACAAG }(C / A)(T / C)(G / C) \text { TGCT (T/A) CA } \\
\text { A (T/C) (A/C) CC (T/C) TCTTCAACT (T/C) (T/C) GAG }\end{array}$ \\
\hline XM_001926775 & similar to glycoprotein $\mathrm{M} 6 \mathrm{~A}$ & 0 & GAAAATGAAAGTGC (T/C) CCTCATCAATGATAT \\
\hline BG610286 & $\begin{array}{l}324863 \text { MARC 1PIG Sus scrofa cDNA } \\
5^{\prime}, \text { mRNA sequence }\end{array}$ & 0 & GATCAGGTGACAAG (C/T) TAACTGGCTACCAAC \\
\hline AY216477 & glutamate-ammonia ligase & $2 e-169$ & GGTGGGCCTTCCA (C/T) TCCTTAGAAGGTTAGA \\
\hline EW345807 & $\begin{array}{l}\text { rplun0127_a19.y1 lun Sus scrofa cDNA } \\
\text { 5', mRNA sequence }\end{array}$ & $6 e-104$ & GATTAAAAGTGATG (G/A) TGGTTTGGGGAGAG \\
\hline CA778985 & $\begin{array}{l}\text { MPL384_11_K21 MPL Sus scrofa cDNA } \\
\text { clone pSPORT1 } 5 \text { ', mRNA sequence }\end{array}$ & 0 & $\begin{array}{l}\text { TTTAGGAAGCA (T/C) TTGATTAAC } \\
\text { ATTTAGTAGTTA (C/A) AGAAATAG }\end{array}$ \\
\hline BG894744 & $\begin{array}{l}\text { 355473 MARC 1PIG Sus scrofa cDNA } \\
5 \text { ', mRNA sequence }\end{array}$ & 0 & $\begin{array}{l}\text { CTGTCCTTGCTCAGT (A/G) GGTTAAAGGATCTGG } \\
\text { AGCTGCAGC (G/A) TAGGTTGCAG(C/A) TGTAGC }\end{array}$ \\
\hline BE234314 & $\begin{array}{l}141142 \text { MARC 1PIG Sus scrofa cDNA } \\
5^{\prime} \text {, mRNA sequence }\end{array}$ & 0 & GGAAATGCTGGTAG (T/C) CGTCAGTATGTCCATT \\
\hline BC146251 & synaptotagmin XI & $1 e-153$ & ATTGCCCTCACCGT (C/A) ATCCTTCTCCGATGTG \\
\hline BC109983 & nuclear transport factor 2 & 0 & $\begin{array}{l}\text { GAGGCCGGGTGAC (A/G) CTCCAGAATGGGAGAC } \\
\text { AAGGAC (T/A) GCAATTCCAGGG (G/A) AAAACTG } \\
\text { GTGGGAGTGGCGC (G/A) GTGCGCTGCTGCTAC }\end{array}$ \\
\hline XM_001254701 & calponin 3 , acidic & 0 & ATCAGAAGCTAACAC (C/T) ACAACCCGTGGACAA \\
\hline XM_001928448 & $\begin{array}{l}\text { similar to Mitogen-activated } \\
\text { protein-binding protein-interacting } \\
\text { protein }\end{array}$ & 0 & $\begin{array}{l}\text { GGGATCAGGTTGTGA (G/A) AGCCCAGGGTTAGGA } \\
\text { GCCACAGGCA (C/T) GCTGCGTCCCAAGGCTTTGA } \\
\text { CTAAGCCAAGC (T/C) AACACTGGAGGTGTCCAGA } \\
\text { GCACCCTGC (C/T) GCTGAATAACGAGGGATCTCT } \\
\text { GCATGTATGCC (T/A) AGGAGACTGTTGGCTTCGG }\end{array}$ \\
\hline AB436774 & MHC class I antigen 2 & 0 & $\begin{array}{l}\text { G (A/G) CCCTGGCCCTGACCGGGACCCAGGCGGGT (T/C) C } \\
\text { CCGCTTC (C/T) TCA (C/T) (C/T) GTCGG (G/C) TACGTG } \\
\text { T (C/T) CGACAGCGACGCCCC (G/A) AAT (C/G) C (G/A) A } \\
\text { (G/A) G (G/A) (A/T) GGAGCCGCGGGCGC (A/C) GTGGA } \\
\text { TA (A/C) AGCAGGAGGGGC (C/A) GGAGTATTGGGATCGGGA } \\
\text { GGA (A/C) ACCGCACAGACTT (A/T) CCGAG (T/G) G } \\
\text { (G/A) (G/A) CCTG (A/C) (A/G) (G/C) AC (C/A) G } \\
\text { CTC (G/T) CGGCTACTACAACCAGAGCGAGGCCGGGTCTCACA } \\
\text { (C/T) TCCAGA (G/C) (A/C) ATGTACGGCTGC (T/G) A } \\
\text { (T/G) TGGG (A/G) CCAGACGGGCTCCTCCTCC (G/A) C } \\
\text { ACA (T/C) GGCGGCTCAGATC (T/A) CCAAGCGCAAGTGGGA }\end{array}$ \\
\hline
\end{tabular}


84 Genomics \& Informatics Vol. 7(2) 65-84, June 2009

Table S1. Continued

GGCGGCC (G/A) ATG (A/T) GGCGGAGCAG (C/G) AGAG GAGCTACCTGCAGGGCC (T/G) GTGTGTGGAGGGGCTCCAGAA GTCACCTTGAGGTGCTGG (A/G) CCCTGGGCTTCTACCCTAAGG A (C/G) CTGGCAGCGGGAGGGCCAGGACCAGAGCCA (A/G) G GACCTTCCAGAAGTGGGC (G/A) GCCCTGGTGGTGCCTCCTGG 